\title{
Multi-attribute group decision making based on Choquet integral under interval-valued intuitionistic fuzzy environment
}

\author{
Jindong Qin ${ }^{1}$, Xinwang Liu ${ }^{1}$, Witold Pedrycz ${ }^{2}$ \\ ${ }^{1}$ School of Economics and Management, Southeast University, \\ Nanjing, 211189, China \\ E-mail: qinjindongseu@126.com,xwliu@seu.edu.cn \\ ${ }^{2}$ Department of Electrical and Computer Engineering, University of Alberta, \\ Edmonton, T6G 2G7, Canada \\ E-mail: wpedrycz@ualberta.ca
}

Received 14 November 2014

Accepted 5 October 2015

\begin{abstract}
In this paper, we propose new methods to represent interdependence among alternative attributes and experts' opinions by constructing Choquet integral using interval-valued intuitionistic fuzzy numbers. In the sequel, we apply these methods to solve the multiple attribute group decision-making (MAGDM) problems under interval-valued intuitionistic fuzzy environment. First, the concept of interval-valued intuitionistic fuzzy Choquet integral is defined, and some elementary properties are studied in detail. Next, an axiomatic system of interval-valued intuitionistic fuzzy measure is established by delivering a series of mathematical proofs. Then, with fuzzy entropy and Shapely-values in game theory, we propose the interval-valued intuitionistic fuzzy measure development methods in order to form the importance measure of attributes and correlation measure of the experts, respectively. Based on the results of theoretical analysis, a new method is proposed to handle the interval-valued intuitionistic fuzzy group decision making problems. A numerical example illustrates the procedure of the proposed methods and verifies the validity and effectiveness of our new proposed methods.
\end{abstract}

Keywords: Interval-valued intuitionistic fuzzy Choquet integral, interval-valued intuitionistic fuzzy sets, interval-valued intuitionistic fuzzy measure, multiple attribute group decision making.

\section{Introduction}

With the increasing complexity and uncertainty of the social-economic environment and various limitations, such as time pressure, lack of knowledge of problem domain, difficulties in information extraction etc., there are numerous uncertain phenomena existing in our daily life. Therefore, in order to better understand the vagueness and uncertainty of the real world and being able to explain it, Zadeh ${ }^{1}$ pro- posed fuzzy set theory in 1960s. Since its inception, the fuzzy set theory has shown convenience as a powerful tool for modeling vagueness and uncertainty in a variety domains, such as economics ${ }^{2,3,4}$, management $5,6,7,8$, artificial intelligence ${ }^{16}$, processing control 10,11 , pattern recognition ${ }^{12}$, decision making 13,14,15,16 etc. In 1980s, Atanassov ${ }^{17,18}$ generalized fuzzy set theory by bringing an idea of intuitionistic fuzzy set (IFS). IFS is characterized by three important notations: membership de-

\footnotetext{
* Corresponding author, Email: xwliu@ seu.edu.cn (X.Liu).
} 
gree, non-membership degree and hesitancy degree. On the basis of these three indexes, IFS can cope with the vagueness and uncertainty characteristics of complex systems. Now, IFS has become an important area of research, and attracted more attention in various fields. For example, $\mathrm{Xu}{ }^{19,20}$ has completed some research in the field of IFS, especially in aggregation operators, developed a series of intuitionistic fuzzy information aggregation operators for aggregating intuitionistic fuzzy information and applied these operators to multiple attribute decision making (MADM) problems. Wang and Liu ${ }^{22}$ proposed the intuitionistic fuzzy aggregation operators realized through Einstein operations and analyzed the relations between these operators and some existing intuitionistic fuzzy aggregation operators. $\mathrm{Li}^{23}$ developed the concept of IFS, and further extended mathematical programming methodology of matrix games with payoffs represented by IFS. Furthermore, some desirable properties were discussed in detail. Farhadinia and Ban ${ }^{24}$ developed some new similarity measures to deal with generalized intuitionistic fuzzy numbers.

However, in many practical situations, there are limitations given various factors, such as a lack of information, uncertainty of the decision making environment, difficulties in information extraction etc. Therefore, it is not easy for decision maker to determine exact values of membership degree, nonmembership degree and hesitancy degree. To overcome these limitations, Atanassov and Gargov ${ }^{18}$ proposed the concept of the interval-valued intuitionistic fuzzy set (IVIFS), in which the attributes are taken the form with interval-valued intuitionistic fuzzy number (IVIFN). Many studies were completed in recent years and various approaches to solve MADM problems under interval-valued intuitionistic fuzzy environment have been developed $^{24,25,26,27,28,29,30,31,32,33,34}$. For example, Farhadinia ${ }^{31}$ generalized recent results for the entropy of interval-valued fuzzy set (IVFS) based on the intuitionistic distance measure and its relationship with the similarity measure, and then applied them to interval-valued intuitionistic fuzzy decision making problem. Liu ${ }^{32}$ developed some geometric aggregation operators based on interval-valued intuition- istic uncertain linguistic variables and some desirable properties were discussed as well. Wang and $\mathrm{Li}^{34}$ proposed a framework to handle multi-attribute group decision making (MAGDM) problems with incomplete pair-wise comparison preference over decision alternatives. Wu and Chiclana ${ }^{33}$ defined interval-valued intuitionistic fuzzy COWA operator and a new score function, then proposed some non-dominance and attitudinal prioritization decision making methods for intuitionistic fuzzy preference relations.

As the most important branch of fuzzy mathematics, fuzzy integrals were originally investigated by Choquet ${ }^{36}$ in 1950s, as a powerful tool for modeling interaction phenomena among various factors. The main difference between the fuzzy integral and the classic integral is that fuzzy integral focuses on non-additive cases while the classical integral only consider additive cases. On the basis of classic sets theory and measure theory, it can be easily proved the fact that the Choquet integral is a special case of classical integral when Choquet integral satisfies the condition of additivity, In this case Choquet integral reduces to the Lebesgue integral. Therefore, the Choquet integral is more general. Compared with the Sugeno integral ${ }^{37}$, which is also known as a fuzzy integral for aggregating discrete sets, Choquet integral exhibits interesting mathematical properties, such as boundary conditions, monotonicity, continuity from below, continuity from above. Therefore, Choquet integral is more suitable to cope with fuzzy uncertainty problems in practical applications. Recently, Choquet integral has been widely used in decision making $35,38,45,46,48$, especially in intuitionistic fuzzy MADM problems. $\mathrm{Xu}{ }^{40} \mathrm{in}-$ vestigated Choquet integral to propose some intuitionistic fuzzy aggregation operators, and then applied them to solve intuitionistic fuzzy multiple attribute decision making problems. Tan and Chen ${ }^{42,43}$ developed an weight solution method based on Choquet integral and further proposed a new Choquet integral-TOPSIS approach to handle MADM problems. Meng and Tang ${ }^{44}$ developed a intervalvalued intuitionistic fuzzy multi-attribute group decision making approach based on cross entropy measure and Choquet integral. Compared with other 
existing methods, this algorithm can not only deal with the relevance among the indexes, but also can cope with dynamic fuzzy systems. Meng et al. ${ }^{45}$ defined some new hybrid Choquet integral aggregation operators, and proposed a method to solve multiple criteria group decision making (MCGDM) based on intuitionistic fuzzy Choquet integral with respect to the generalized $\lambda$-Shapely index. Wu et al. ${ }^{47}$ presented a detailed discussion on the integration properties of the interval intuitionistic fuzzy conjugate Choquet integral, and applied these theoretical analysis results to software development risks decision making. In addition, some authors have also applied Choquet integral to other fields, such as hesitant fuzzy set ${ }^{49}$, game theory ${ }^{50}$, neural networks ${ }^{51,52}$, statistical learning ${ }^{53}$, and combinatorial optimization $54,55,56$.

According to the previous overview, intuitionistic fuzzy Choquet integral and its property has been widely used in decision making problems. However, there has been a limited research on Choquet integral under interval-valued intuitionistic fuzzy decision making environment in the literatures. When Choquet integral is used to handle MADM problems under interval-valued intuitionistic fuzzy environment, the most challenging difficulty is how to determine fuzzy measure with an effective and accurate method. For an MADM problem which contains $n$ attributes, we should determine $2^{n}-2$ elements of fuzzy measures in decision making process. When the problem dimensionality increases, the computational complexity increases rapidly. So far, in the current literature $42,43,44,45,57,58,59$, the fuzzy measures are usually provided by decision makers (DMs) in advance, which maybe difficult to the DMs and is closely related the subjective preferences of the DMs, or can even lead to inconsistent results. If the fuzzy measures can be determined from the problem formulation or the decision maker's known information directly, the decision making model solution can be more reliable and objective than the current ones. Therefore, how to determine the fuzzy measure based on the expert decision making information becomes a very important issue both in theory and practice. The motivation of this study is to develop a new method of constructing fuzzy measures based on interval-valued intuitionistic information and apply them to the group decision making problems.

The remainder of this paper is organized as follows. In Section 2, we briefly introduce some basic concepts and related operational laws of intervalvalued intuitionistic fuzzy set (IVIFS) and the Choquet integral. In Section 3, we give the definition of interval-valued intuitionistic fuzzy measure with Choquet integral and propose some useful theoretical background. In Section 4, we develop new methods to determine fuzzy measure in real-world decision marking situations, by establishing intervalvalued intuitionistic fuzzy measure to determine the measure of expert weights and attribute weights, and strict mathematical proof process is given. In Section 5, a new approach based on interval-valued intuitionistic fuzzy Choquet integral is proposed to solve MAGDM problems. In Section 6, an illustrative example is provided to illustrate the proposed method. Finally, we come up with some conclusions and point out the future research in Section 7.

\section{Preliminaries}

In this section, we briefly review some basic concepts of interval-valued intuitionistic fuzzy set (IVIFS) and fuzzy measure, which are extended to interval-valued intuitionistic fuzzy measure in next Section 3.

\subsection{Interval-valued intuitionistic fuzzy set}

Definition $1^{18}$ Let $X$ be a universe of discourse. Then

$$
A=\left\{\left\langle x,\left(\left[\mu_{A}^{L}(x), \mu_{A}^{R}(x)\right],\left[v_{A}^{L}(x), v_{A}^{R}(x)\right]\right) \mid x \in X\right\rangle\right\}
$$

is an IVIFS, where $\mu_{A}(x)=\left[\mu_{A}^{L}(x), \mu_{A}^{R}(x)\right], v_{A}(x)=$ $\left[v_{A}^{L}(x), v_{A}^{R}(x)\right]$ is called as interval membership degree and non-membership degree of $x$. The following two conditions are satisfied:

(1) For all $x \in X,\left[\mu_{A}^{L}(x), \mu_{A}^{R}(x)\right] \subseteq[0,1]$, and $\left[v_{A}^{L}(x), v_{A}^{R}(x)\right] \subseteq[0,1]$;

(2) $\forall x \in X, 0 \leqslant \mu_{A}^{R}(x)+v_{A}^{R}(x) \leqslant 1$. 
In particular, if $\mu_{A}^{L}(x)=\mu_{A}^{R}(x), v_{A}^{L}(x)=v_{A}^{R}(x)$, for each $x \in X$, then the IVIFS is reduced to IFS.

For convenience, $\mathrm{Xu} 20$ called $\alpha=$ $\left(\left[a^{L}, a^{R}\right],\left[b^{L}, b^{R}\right]\right)$ an interval-valued intuitionistic fuzzy number (IVIFN), where $\left[a^{L}, a^{R}\right] \subseteq$ $[0,1],\left[b^{L}, b^{R}\right] \subseteq[0,1], a^{R}+b^{b} \leqslant 1$, and let $\Theta$ be the set of all IVIFNs. Obviously, $\alpha^{+}=([1,1],[0,0])$ is the largest IVIFN, and $\alpha^{-}=([0,0],[1,1])$ is the smallest IVIFN.

Definition $2{ }^{20}$ Let $\alpha=\left(\left[a^{L}, a^{R}\right],\left[b^{L}, b^{R}\right]\right), \beta=$ $\left(\left[c^{L}, c^{R}\right],\left[d^{L}, d^{R}\right]\right)$ be two IVIFNs. The operational laws are shown as follows:

(1) $\alpha \oplus \beta=\left(\left[a^{L}+c^{L}-a^{L} c^{L}, a^{R}+c^{R}-\right.\right.$ $\left.\left.a^{R} c^{R}\right],\left[b^{L} d^{L}, b^{R} d^{R}\right]\right) ;$

(2) $\alpha \otimes \beta=\left(\left[a^{L} c^{L}, a^{R} c^{R}\right],\left[b^{L}+d^{L}-b^{L} d^{L}, b^{R}+\right.\right.$ $\left.\left.d^{R}-b^{R} d^{R}\right]\right)$;

(3) $\alpha^{C}=\left(\left[b^{L}, b^{R}\right],\left[a^{L}, a^{R}\right]\right)$;

(4) $a^{\lambda}=\left(\left[\left(a^{L}\right)^{\lambda},\left(a^{R}\right)^{\lambda}\right],\left[1-\left(1-b^{L}\right)^{\lambda}, 1-\right.\right.$ $\left.\left.\left(1-b^{R}\right)^{\lambda}\right]\right), \lambda>0$.

Definition $3{ }^{19}$ Let $\alpha=\left(\left[a^{L}, a^{R}\right],\left[b^{L}, b^{R}\right]\right)$ be an IVIFN. Then the score function of $\alpha$ is defined as follows:

$$
s(\alpha)=\frac{a^{L}+a^{R}-b^{L}-b^{R}}{2}
$$

where $s(\alpha) \in[-1,1]$. Obviously, the greater $s(\alpha)$ is, the larger IVIFN $\alpha$ becomes.

Definition $4{ }^{21}$ Let $A=\left\{\left\langle x_{i},\left(\left[a_{i}^{L}, a_{i}^{R}\right],\left[b_{i}^{L}, b_{i}^{R}\right]\right) \mid x \in X\right\rangle\right\}$ $(i=1,2, \cdots, n)$ be a discrete IVIFS. The entropy of $A$ is defined in the following form:

$$
E(A)=\frac{1}{n} \sum_{i=1}^{n} \cos \frac{\left|b_{i}^{L}-a_{i}^{L}\right|+\left|b_{i}^{R}-a_{i}^{R}\right|}{2\left(\pi_{i}^{L}+\pi_{i}^{R}+2\right)} \pi
$$

The interval-valued intuitionistic fuzzy entropy satisfies the following properties:

(1) $E(A)=0$ if and only if $a_{i}^{L}=a_{i}^{R}=0, b_{i}^{L}=b_{i}^{R}=1$ or $a_{i}^{L}=a_{i}^{R}=1, b_{i}^{L}=b_{i}^{R}=0 ;$

(2) $E(A)=1$ if and only if $\left[a_{i}^{L}, a_{i}^{R}\right]=\left[b_{i}^{L}, b_{i}^{R}\right]$, for any $x_{i} \in X$;

(3) $E(A)=E\left(A^{c}\right)$;
(4) $E(A) \leqslant E(B), \forall x_{i} \in X$, if $a_{B}^{L} \leqslant b_{B}^{L}, a_{B}^{R} \leqslant b_{B}^{R}$, then $a_{A}^{L} \leqslant a_{B}^{L}, a_{A}^{R} \leqslant a_{B}^{R}, b_{A}^{L} \geqslant b_{B}^{L}, b_{A}^{R} \geqslant b_{B}^{R}$ or $a_{B}^{L} \geqslant b_{B}^{L}, a_{B}^{R} \geqslant b_{B}^{R}$, then $a_{A}^{L} \geqslant a_{B}^{L}, a_{A}^{R} \geqslant a_{B}^{R}, b_{A}^{L} \leqslant$ $b_{B}^{L}, b_{A}^{R} \leqslant b_{B}^{R}$.

\subsection{Fuzzy measure and Choquet integral}

Definition $5^{60}$ Let $X$ be a universe of discourse, $P(X)$ be the power set of $X$. A fuzzy measure on $X$ is a mapping $\mu: P(X) \mapsto[0,1]$ which satisfies the following properties:

(1) $\mu(\phi)=0, \mu(X)=1$ (boundary conditions);

(2) if $A \subseteq B$ implies $\mu(A) \leqslant \mu(B)$, for all $A, B \subseteq$ $X$,(monotonicity);

(3) $\mu(A+B)=\mu(A)+\mu(B)+\lambda \mu(A) \mu(B)$, where $A, B \subseteq P(X)$ and $A \cap B=\phi, \lambda \geqslant-1$;

(4) $\mu\left(\bigcup_{n=1}^{n} A_{n}\right)=\lim _{n \rightarrow \infty} \mu\left(A_{n}\right)$, whenever $A_{n} \subseteq$ $A_{n+1}, A_{n} \in X, n \in N$ (continuity from below);

(5) $\mu\left(\bigcap_{n=1}^{\infty} A_{n}\right)=\lim _{n \rightarrow \infty} \mu\left(A_{n}\right)$, whenever $A_{n} \supseteq$ $A_{n+1}, A_{n} \in X, n \in N$ (continuity from above).

Definition $6{ }^{37}$ Let $X=\left\{x_{1}, x_{2}, \cdots, x_{n}\right\}$ be a universe of discourse, and $X=\bigcup_{i=1}^{n} x_{i}$, then $\lambda-f u z z y$ measure $\mu$ satisfies following condition:

$$
\mu(X)= \begin{cases}\frac{1}{\lambda}\left(\prod_{i=1}^{n}\left[1+\lambda \mu\left(x_{i}\right)\right]-1\right) & \text { if } \lambda \neq 0 \\ \sum_{i=1}^{n} \mu\left(x_{i}\right) & \text { if } \lambda=0\end{cases}
$$

where $\lambda>-1$. From Eq.(4), the value of $\lambda$ can be uniquely determined by setting $\mu(X)=1$, which is equivalent by solving the following equation:

$$
\lambda+1=\prod_{i=1}^{n}\left(1+\lambda \mu\left(x_{i}\right)\right)
$$

Definition $7{ }^{35}$ Let $\mu$ be a fuzzy measure on $X, f$ is a nonnegative, real-valued, and measurable function. Then the Choquet integral is defined in the form:

$$
\text { (c) } \int f d \mu=\int_{0}^{\infty} \mu\left\{F_{\alpha}\right\} d l
$$

where $F_{\alpha}=\{x \mid f(x) \geqslant \alpha\}$ denotes the $\alpha$-cut of the function. When $X=\left\{x_{1}, x_{2}, \cdots, x_{n}\right\}$ be a discrete 
set, the discrete form of Choquet integral with respect to a fuzzy measure $\mu$ is defined as:

(c) $\int f \circ d \mu=\sum_{i=1}^{n}\left(f\left(x_{i}^{*}\right)-f\left(x_{i-1}^{*}\right)\right) \mu\left(\left\{x_{i}^{*}, x_{i+1}^{*}, \cdots, x_{n}^{*}\right\}\right)$

Where $A^{*}=\left\{x_{1}^{*}, x_{2}^{*}, \cdots, x_{n}^{*}\right\}$ is a monotonic nondecrease permutation of $X=\left\{x_{1}, x_{2}, \cdots, x_{n}\right\}$ such that $f\left(x_{1}^{*}\right) \geqslant f\left(x_{2}^{*}\right) \geqslant \cdots \geqslant f\left(x_{n}^{*}\right)$ and $A_{n+1}^{*}=\emptyset$.

\section{Interval-valued intuitionistic fuzzy Choquet integral}

In this section, we investigate the definition of the interval-valued intuitionistic fuzzy measure and the interval-valued intuitionistic fuzzy Choquet integral with such fuzzy measure. Some properties and characteristics of them are proposed and discussed in detail.

Definition 8 Let $X$ be a universe of discourse, and $(X, \Sigma, \mu)$ be an interval intuitionistic fuzzy measure space, $\Sigma$ is a $\sigma$ - algebra and $\mu=$ $\left(\left[\mu_{1}, \mu_{2}\right],\left[\mu_{3}, \mu_{4}\right]\right)$, where fuzzy measure $\mu$ satisfies the following properties:

(1) $\mu(\phi)=\left(\left[\mu_{1}(\phi), \mu_{2}(\phi)\right],\left[\mu_{3}(\phi), \mu_{4}(\phi)\right]\right)=$ $([0,0],[1,1])$

(2) $\mu(X)=\left(\left[\mu_{1}(X), \mu_{2}(X)\right],\left[\mu_{3}(X), \mu_{4}(X)\right]\right)=$ $([1,1],[0,0])$;

(3) If $E, F \in \Sigma$, and $E \subseteq F$, then $\mu_{1}(E) \leqslant$ $\mu_{1}(F), \mu_{2}(E) \leqslant \mu_{2}(F)$ and $\mu_{3}(E) \geqslant$ $\mu_{3}(F), \mu_{4}(E) \geqslant \mu_{4}(F)$;

(4) For any $E \in \Sigma, \mu_{2}(E)+\mu_{4}(E) \leqslant 1$.

Especially, when $\mu_{1}(E)=\mu_{2}(E), \mu_{3}(E)=$ $\mu_{4}(E)$, for any $E \in \Sigma$, the interval-valued intuitionistic fuzzy measure reduces to the commonly encountered intuitionistic fuzzy measure.

Based on the decomposition theorem for the original Choquet integral and the extended intuitionistic fuzzy Choquet integral, the interval-valued intuitionistic fuzzy Choquet integral of $f$ with respect to $\mu$ is defined as:

$$
\left.\begin{array}{r}
(c) \int f \circ d \mu=\left(\left[\int a_{i}^{L} \circ d \mu_{1}, \int a_{i}^{R} \circ d \mu_{2}\right],\right. \\
{\left[\int \overline{b_{i}^{L}} \circ d \overline{\mu_{3}}, \int \overline{b_{i}^{R}} \circ d \overline{\mu_{4}}\right]}
\end{array}\right)
$$

where $f=\left(\left[a_{i}^{L}, a_{i}^{R}\right],\left[b_{i}^{L}, b_{i}^{R}\right]\right), \mu=\left(\left[\mu_{1}, \mu_{2}\right],\left[\mu_{3}, \mu_{4}\right]\right)$. For any set $A \in \Sigma$, which satisfies $\mu_{1}(A) \leqslant$ $\mu_{2}(A), \mu_{3}(A) \leqslant \mu_{4}(A), \mu_{2}(A)+\mu_{4}(A) \leqslant 1$, and "" denotes the dual operation, i.e.,

$$
\bar{f}(x)=1-f(x) \quad \bar{\mu}(A)=1-\mu(A)
$$

Theorem 1 Let $\mu$ be an interval-valued intuitionistic fuzzy measure defined on $X$, and $A$ is an intervalvalued intuitionistic fuzzy set on $P(X)$. Then the interval-valued intuitionistic fuzzy Choquet integral with respect to $\mu$ is also an IVIFN.

Proof. Based on the definition of IVIFS, we have $a^{L} \leqslant a^{R}, b^{L} \leqslant b^{R}$. According to the monotonicity of fuzzy measure, we can prove that $\int a_{i}^{L} d \mu_{1} \leqslant \int a_{i}^{R} d \mu_{1} \leqslant \int a_{i}^{R} d \mu_{2}$ and $\overline{\int \overline{b_{i}^{L}} d \overline{\mu_{3}}}=1-$ $\int \overline{b_{i}^{L}} d \overline{\mu_{3}}=1-\int\left(1-b_{i}^{L}\right) d \overline{\mu_{3}} \leqslant 1-\int\left(1-b_{i}^{R}\right) d \overline{\mu_{3}} \leqslant$ $1-\int\left(1-b_{i}^{R}\right) d \overline{\mu_{4}}=1-\int \overline{b_{i}^{R}} d \overline{\mu_{4}}=\overline{\int \overline{b_{i}^{R}} d \overline{\mu_{4}}}$. Thus, Eq. (8) satisfies the partial order of the IVIFS.

For convenience, we denote $f_{1}=a_{i}^{L}, f_{2}=$ $a_{i}^{R}, f_{3}=b_{i}^{L}, f_{4}=b_{i}^{R}$ in the following proof process. Based on the definition of discrete Choquet integral described in Section 2, we have:

$$
\begin{aligned}
& \text { (c) } \int f_{1} \circ \mu_{1}=\int \mu_{1}\left(f_{1}(x) \geqslant \alpha_{1}\right) d \alpha_{1} \\
& \text { (c) } \int f_{2} \circ \mu_{2}=\int \mu_{2}\left(f_{2}(x) \geqslant \alpha_{2}\right) d \alpha_{2}
\end{aligned}
$$

So we only need to prove the following relationships:

$$
\begin{aligned}
& \text { (c) } \int f_{3} \circ \mu_{3}=\int \mu_{3}\left(f_{3}(x) \leqslant \alpha_{3}\right) d \alpha_{3} \\
& \text { (c) } \int f_{4} \circ \mu_{4}=\int \mu_{4}\left(f_{4}(x) \leqslant \alpha_{4}\right) d \alpha_{4}
\end{aligned}
$$


Based on Eq. (10), we have

$$
\begin{aligned}
\overline{\int \overline{f_{3}} \circ \overline{\mu_{3}}} & =1-\int_{0}^{1} \overline{\mu_{3}}\left(\overline{f_{3}}(x) \geqslant \alpha_{3}\right) d \alpha_{3} \\
& =1-\int_{0}^{1}\left[1-\overline{\mu_{3}}\left(\overline{f_{3}}(x) \geqslant \alpha_{3}\right)\right] d \alpha_{3} \\
& =\int_{0}^{1} \overline{\mu_{3}}\left(\overline{f_{3}}(x) \geqslant \alpha_{3}\right) d \alpha_{3} \\
& =\int_{0}^{1}\left(1-\mu_{3}\right)\left(f_{3}(x) \leqslant 1-\alpha_{3}\right) d \alpha_{3} \\
& =1-\int_{0}^{1} \mu_{3}\left(f_{3}(x) \leqslant 1-\alpha_{3}\right) d \alpha_{3} \\
& =\int_{0}^{1} \mu_{3}\left(f_{3}(x) \leqslant \alpha_{3}\right) d \alpha_{3}
\end{aligned}
$$

In a similar way, it is easy to prove that $(c) \int f_{4}$ 。 $\mu_{4}=\int \mu_{4}\left(f_{4}(x) \leqslant \alpha_{4}\right) d \alpha_{4}$ holds.

Next, Based on the definition of interval intuitionistic fuzzy sets described in Section 2, we only need to prove that the following inequality holds:

$$
\text { (c) } \int f_{2} \circ \mu_{2}+(c) \overline{\int \bar{f}_{4} \circ \overline{\mu_{4}}} \leqslant 1
$$

Since $f_{2}+f_{4} \leqslant 1, \mu_{2}+\mu_{4} \leqslant 1$, based on the monotonicity of Choquet integral, the following inequality ensues:

$$
\text { (c) } \int \bar{f}_{4} \circ \overline{\mu_{4}} \geqslant(c) \int f_{2} \circ \overline{\mu_{4}} \geqslant(c) \int f_{2} \circ \mu_{2}
$$

Then

$$
\begin{gathered}
(c) \int f_{2} \circ \mu_{2}+(c) \overline{\int \bar{f}_{4} \circ \overline{\mu_{4}}}=(c) \int f_{2} \circ \mu_{2}+1- \\
(c) \int \bar{f}_{4} \circ \overline{\mu_{4}} \leqslant(c) \int f_{2} \circ \mu_{2}+1-(c) \int f_{2} \circ \mu_{2}=1
\end{gathered}
$$

The proof has been completed.

Theorem 2 Let $X=\left\{x_{1}, x_{2}, \cdots, x_{n}\right\}$ be the universe of discourse, then the interval-valued intuitionistic fuzzy Choquet integral of $f$ with respect to $\mu$ can be expressed as the following form:

$$
\begin{aligned}
(c) \int f^{\circ} d \mu= & \left(\left[\begin{array}{c}
\sum_{i=1}^{n} a_{i}^{L}\left(x_{i}\right)\left(\mu_{1}\left(A_{i}\right)-\mu_{1}\left(A_{i+1}\right)\right), \\
\sum_{i=1}^{n} a_{i}^{R}\left(x_{i}\right)\left(\mu_{2}\left(A_{i}\right)-\mu_{2}\left(A_{i+1}\right)\right)
\end{array}\right],\right. \\
& {\left.\left[\begin{array}{c}
\sum_{i=1}^{n} b_{i}^{L}\left(x_{i}\right)\left(\mu_{1}\left(B_{i+1}\right)-\mu_{1}\left(B_{i}\right)\right), \\
\sum_{i=1}^{n} b_{i}^{R}\left(x_{i}\right)\left(\mu_{4}\left(B_{i+1}\right)-\mu_{4}\left(B_{i}\right)\right)
\end{array}\right]\right) }
\end{aligned}
$$

where

$$
\begin{aligned}
& A_{i}=\left\{x_{i}, \cdots, x_{n} \mid\left(a^{L}+a^{R}\right)\left(x_{i+1}\right) \geqslant\left(a^{L}+a^{R}\right)\left(x_{i}\right)\right\} \\
& \text { and } \\
& B_{i}=\left\{x_{i}, \cdots, x_{n} \mid\left(b^{L}+b^{R}\right)\left(x_{i+1}\right) \leqslant\left(b^{L}+b^{R}\right)\left(x_{i}\right)\right\} \\
& (i=1,2, \cdots, n)
\end{aligned}
$$

Proof. Based on the definition of Choquet integral described in Section 2, it can be easily shown that the following two relationships are satisfied:

$$
\begin{aligned}
& \text { (c) } \int f_{1} \circ d \mu_{1}=\sum_{i=1}^{n} f_{1}\left(x_{i}\right)\left(\mu_{1}\left(A_{i}\right)-\mu_{1}\left(A_{i+1}\right)\right) \\
& =\sum_{i=1}^{n} a_{i}^{L}\left(x_{i}\right)\left(\mu_{1}\left(A_{i}\right)-\mu_{1}\left(A_{i+1}\right)\right) \\
& \text { (c) } \int f_{2} \circ d \mu_{2}=\sum_{i=1}^{n} f_{2}\left(x_{i}\right)\left(\mu_{2}\left(A_{i}\right)-\mu_{2}\left(A_{i+1}\right)\right) \\
& =\sum_{i=1}^{n} a_{i}^{R}\left(x_{i}\right)\left(\mu_{2}\left(A_{i}\right)-\mu_{2}\left(A_{i+1}\right)\right)
\end{aligned}
$$

Thus, we should only prove the following formulas:

$$
\begin{aligned}
(c) \overline{\int \bar{f}_{3} \circ d \bar{\mu}_{3}} & =\sum_{i=1}^{n} \bar{f}_{3}\left(x_{i}\right)\left(\bar{\mu}_{3}\left(B_{i+1}\right)-\bar{\mu}_{3}\left(B_{i}\right)\right) \\
& =\sum_{i=1}^{n} b_{i}^{L}\left(x_{i}\right)\left(\mu_{3}\left(B_{i+1}\right)-\mu_{3}\left(B_{i}\right)\right) \\
(c) \overline{\int \bar{f}_{4} \circ d \bar{\mu}_{4}} & =\sum_{i=1}^{n} \bar{f}_{4}\left(x_{i}\right)\left(\bar{\mu}_{4}\left(B_{i+1}\right)-\bar{\mu}_{4}\left(B_{i}\right)\right) \\
& =\sum_{i=1}^{n} b_{i}^{R}\left(x_{i}\right)\left(\mu_{4}\left(B_{i+1}\right)-\mu_{4}\left(B_{i}\right)\right)
\end{aligned}
$$


Based on Eqs. $(9,10,12)$, we obtain

$$
\begin{aligned}
& (c) \overline{\int \overline{b^{L}} \circ d \overline{\mu_{3}}} \\
& =\overline{\int \overline{f_{3}} \circ d \overline{\mu_{3}}} \\
& =1-\int \overline{f_{3}} \circ d \overline{\mu_{3}} \\
& =1-\sum_{i=1}^{n} \overline{f_{3}}\left(x_{i}\right)\left[\overline{\mu_{3}}\left(B_{i}\right)-\overline{\mu_{3}}\left(B_{i+1}\right)\right] \\
& =1-\sum_{i=1}^{n} \overline{f_{3}}\left(x_{i}\right)\left[\left(1-\mu_{3}\left(B_{i}\right)\right)-\left(1-\mu_{3}\left(B_{i+1}\right)\right)\right] \\
& =\sum_{i=1}^{n}\left(\mu_{3}\left(B_{i+1}\right)-\mu_{3}\left(B_{i}\right)\right)-\sum_{i=1}^{n} \overline{f_{3}}\left(x_{i}\right)\left(\mu_{3}\left(B_{i+1}\right)-\mu_{3}\left(B_{i}\right)\right) \\
& =\sum_{i=1}^{n}\left(\mu_{3}\left(B_{i+1}\right)-\mu_{3}\left(B_{i}\right)\right)\left(1-\overline{f_{3}}\left(x_{i}\right)\right) \\
& =\sum_{i=1}^{n} f_{3}\left(x_{i}\right)\left(\mu_{3}\left(B_{i+1}\right)-\mu_{3}\left(B_{i}\right)\right)
\end{aligned}
$$

Similarly, it is easy to verify that the following formula holds:

$$
\text { (c) } \overline{\int \overline{b^{R}} \circ d \overline{\mu_{4}}}=\sum_{i=1}^{n} b_{i}^{R}\left(x_{i}\right)\left(\mu_{4}\left(B_{i+1}\right)-\mu_{4}\left(B_{i}\right)\right)
$$

Thus, the proof has been completed.

In what follows, we investigate two useful theorems of the interval-valued intuitionistic fuzzy Choquet integral proposed above.

Theorem 3 Let $X$ be a universe of discourse, $f=$ $\left(\left[f_{1}, f_{2}\right],\left[f_{3} . f_{4}\right]\right)$ be an interval intuitionistic fuzzyvalued function on $X . \overline{0}=([0,0],[1,1])$ and $\overline{1}=$ $([1,1],[0,0])$ are the smallest and largest interval intuitionistic fuzzy-valued function, respectively. Then

1. (Idempotency). $c(\overline{0}, \overline{0}, \cdots, \overline{0})=\overline{0}, c(\overline{1}, \overline{1}, \cdots, \overline{1})=$ $\overline{1}, c(\bar{f}, \bar{f}, \cdots, \bar{f})=\bar{f}$;

2. (Boundary). $\min \left(f^{(1)}, f^{(2)}, \cdots, f^{(n)}\right) \leqslant$ $c\left(f^{(1)}, f^{(2)} \cdots, f^{(n)}\right) \leqslant \max \left(f^{(1)}, f^{(2)}, \cdots, f^{(n)}\right) ;$

3. (Monotonicity). If $f^{(k)} \leqslant f^{\left(k^{*}\right)}$, then $c\left(f^{(1)}\right.$, $\left.\cdots, f^{(k)}, \cdots, f^{(n)}\right) \leqslant c\left(f^{(1)}, \cdots, f^{\left(k^{*}\right)}, \cdots, f^{(n)}\right)$.

\section{Proof.}

(1) Based on the definition of Choquet integral, we have

$$
\begin{aligned}
& (c)(\overline{0}, \overline{0}, \cdots, \overline{0})=\overline{0} \times \sum_{i=1}^{n}\left(\mu\left(x_{i}\right)-\mu\left(x_{i+1}\right)\right)=\overline{0} \times 1=\overline{0} \\
& (c)(\overline{1}, \overline{1}, \cdots, \overline{1})=\overline{1} \times \sum_{i=1}^{n}\left(\mu\left(x_{i}\right)-\mu\left(x_{i+1}\right)\right)=\overline{1} \times 1=\overline{1} \\
& (c)(f, f, \cdots, f)=f \times \sum_{i=1}^{n}\left(\mu\left(x_{i}\right)-\mu\left(x_{i+1}\right)\right)=f \times 1=f
\end{aligned}
$$

(2) Based on Theorems 1 and 2, we obtain $\quad(c)\left(\min _{1 \leqslant i \leqslant n} f^{(i)}, \cdots, \min _{1 \leqslant i \leqslant n} f^{(i)}\right) \leqslant$ $(c)\left(f^{(1)}, \cdots, f^{(n)}\right) \leqslant(c)\left(\max _{1 \leqslant i \leqslant n} f^{(i)}, \cdots, \max _{1 \leqslant i \leqslant n} f^{(i)}\right)$. Then according to the property of idempotency, we have $(c)\left(\min _{1 \leqslant i \leqslant n} f^{(i)}, \cdots, \min _{1 \leqslant i \leqslant n} f^{(i)}\right)=$ $\min _{1 \leqslant i \leqslant n} f^{(i)}$ and $(c)\left(\max _{1 \leqslant i \leqslant n} f^{(i)}, \cdots, \max _{1 \leqslant i \leqslant n} f^{(i)}\right)=$ $\max _{1 \leqslant i \leqslant n} f^{(i)}$. Therefore, $\min \left(f^{(1)}, f^{(2)}, \cdots, f^{(n)}\right) \leqslant$ $(c)\left(f^{(1)}, f^{(2)}, \cdots, f^{(n)}\right) \leqslant \max \left(f^{(1)}, f^{(2)}, \cdots, f^{(n)}\right)$.

(3) Based on the monotonicity of fuzzy integral and Theorem 2, the conclusion directly ensues.

which completes the proof of Theorem 3.

Theorem 4 Let $X$ be a universe of discourse, $P(X)$ is a power set of $X$, set function $\mu$ is an intervalvalued intuitionistic fuzzy measure on $X$, let $f=$ $\left(\left[f_{1}, f_{2}\right],\left[f_{3}, f_{4}\right]\right)$ be an interval-valued intuitionistic fuzzy-valued function $X . \overline{0}=([0,0],[1,1])$ and $\overline{1}=$ $([1,1],[0,0])$ are the smallest and largest intervalvalued intuitionistic fuzzy measure, respectively. Then

1. (c) $\int f \circ \overline{1}=\max _{x \in X} f$;

2. (c) $\int f \circ \overline{0}=\min _{x \in X} f$;

3. For any interval intuitionistic fuzzy measure $\mu$ on $X, \min _{x \in X} f \leqslant(c) \int f \circ \mu \leqslant \max _{x \in X} f$.

\section{Proof.}

(1) For any $\alpha=\left(\left[\alpha_{1}, \alpha_{2}\right],\left[\alpha_{3}, \alpha_{4}\right]\right) \in X$, based on the definition of interval intuitionistic fuzzy 
Choquet integral described in Section 2, we have

$$
\begin{aligned}
& \text { (c) } \int f \circ \mu \\
& =\left(\begin{array}{l}
{\left[\int_{0}^{1} \mu_{1}\left(f_{1} \geqslant \alpha_{1}\right) d \alpha_{1}, \int_{0}^{1} \mu_{2}\left(f_{2} \geqslant \alpha_{2}\right) d \alpha_{2}\right],} \\
{\left[\int_{0}^{1} \mu_{3}\left(f_{3} \leqslant \alpha_{3}\right) d \alpha_{3}, \int_{0}^{1} \mu_{4}\left(f_{4} \leqslant \alpha_{4}\right) d \alpha_{4}\right]}
\end{array}\right)
\end{aligned}
$$

where $\{f \geqslant \alpha\}=\{x \in X \mid f(x) \geqslant \alpha\}=\{x \in$ $X \mid f_{1}(x) \geqslant \alpha_{1} \cap f_{2}(x) \geqslant \alpha_{2} \cap f_{3}(x) \leqslant \alpha_{3} \cap$ $\left.f_{4}(x) \leqslant \alpha_{4}\right\}$. When $\alpha \geqslant \beta$, then $\{x \mid f \geqslant$ $\alpha\} \subseteq\{x \mid f \geqslant \beta\}$, which implies $\mu\{x \mid f \geqslant \alpha\} \leqslant$ $\mu\{x \mid f \geqslant \beta\}$.

If $\mu\{x \mid f \geqslant \alpha\}=\overline{1} \Rightarrow \mu\{x \mid f \geqslant \beta\}=\overline{1}$. Then,

(c) $\int f \circ \mu$
$=\left(\begin{array}{c}{\left[\int_{0}^{1} \mu_{1}\left(f_{1} \geqslant \alpha_{1}\right) d \alpha_{1}, \int_{0}^{1} \mu_{2}\left(f_{2} \geqslant \alpha_{2}\right) d \alpha_{2}\right],} \\ {\left[\int_{0}^{1} \mu_{3}\left(f_{3} \leqslant \alpha_{3}\right) d \alpha_{3}, \int_{0}^{1} \mu_{4}\left(f_{4} \leqslant \alpha_{4}\right) d \alpha_{4}\right]}\end{array}\right)$

(c) $\int f \circ \overline{1}$

$$
\begin{aligned}
& =\sup _{\mu(f \geqslant \alpha)=\overline{1}}\left(\left[\begin{array}{c}
\int_{0}^{1} \mu_{1}\left(f_{1} \geqslant \beta_{1}\right) d \beta_{1}, \\
\int_{0}^{1} \mu_{2}\left(f_{2} \geqslant \beta_{2}\right) d \beta_{2}
\end{array}\right], \quad\left[\begin{array}{c}
\int_{0}^{1} \mu_{3}\left(f_{3} \leqslant \beta_{3}\right) d \beta_{3}, \\
\int_{0}^{1} \mu_{4}\left(f_{4} \leqslant \beta_{4}\right) d \beta_{4}
\end{array}\right]\right) \\
& =\sup _{\mu(f \geqslant \alpha)=\overline{1}}\left(\left[\alpha_{1}, \alpha_{2}\right],\left[\alpha_{3}, \alpha_{4}\right]\right) \\
& =\sup _{\mu(f \geqslant \alpha)=\overline{1}} \alpha \leqslant \sup _{\mu(f \geqslant \alpha)=\overline{1} \mu(f \geqslant \alpha)=\overline{1}} f \\
& \leqslant \sup \quad \sup f=\max f
\end{aligned}
$$

(2) The proof of property 2 is similar to property 1 , omitted.

(3) Based on the monotonicity of fuzzy measure, we have $\overline{0} \leqslant \mu \leqslant \overline{1}$, so we can easily prove the following inequality

$$
\text { (c) } \int f \circ \overline{0} \leqslant(c) \int f \circ \mu \leqslant(c) \int f \circ \overline{1}
$$

According to Theorem 3, we have $\min _{x \in X} f \leqslant$ (c) $\int f \circ \mu \leqslant \max _{x \in X} f$.

which completes the proof of Theorem 4 .

\section{A new method to determine fuzzy measure under interval-valued intuitionistic fuzzy environment}

In most real-world decision making situations, where the measure of attributes usually does not satisfy the condition of non-additive property. However, there exist some cases where the attributes may interact. Therefore, how to derive the fuzzy measures to reflect the relationship of attributes becomes a vital problem in such practical problems. From the current literature $57,58,59$, we can only find research on the determination of the fuzzy measures with the decision makers assignment directly, which not only raises difficulties for large dimensional problems and may also lead to information losses, distortion and inconsistencies. Here, we propose a new method to determine the fuzzy measures from the known information, which can avoid the shortcomings of the current direct assignment methods.

For convenience, we first introduce some new notations. Let $D=\left\{D_{1}, D_{2}, \cdots, D_{p}\right\}$ be the set of experts, where $D_{i}$ indicates the i-th expert, and $E_{i}$ indicates the average interval-valued intuitionistic fuzzy entropy provided by $D_{i}$, which is calculated by Eq. (3).

\subsection{Experts importance measure based on interval-valued intuitionistic fuzzy entropy}

Definition 9 Let $\mu\left(D_{i}\right)$ denote the importance measure of expert $D_{i}$, and $\mu\left(D_{i_{1}}, D_{i_{2}}, \cdots, D_{i_{n}}\right)$ denotes the joint absolute importance measure of experts $D_{i_{1}}, D_{i_{2}}, \cdots, D_{i_{n}}$, the experts absolute importance measure can be defined as:

$$
\begin{gathered}
\mu\left(D_{i}\right)=1-E_{i} \\
\mu\left(D_{i}, D_{j}\right)=1-E\left(E_{i} \cap E_{j}\right) \\
\mu\left(D_{i_{1}}, D_{i_{2}}, \cdots, D_{i_{n}}\right)=1-E\left(E_{i_{1}} \cap E_{i_{2}} \cap \cdots \cap E_{i_{n}}\right)
\end{gathered}
$$

where $\left\{i_{1}, i_{2}, \cdots, i_{n}\right\}$ is a subset of $\{1,2, \cdots, p\}, E_{k i j}$ is the interval-valued intuitionistic fuzzy entropy provided by expert $D_{k}$ for the alternative $A_{i}$ with respect to the attribute $C_{j}$. It should be noted that $E\left(E_{i_{1}} \cap E_{i_{2}} \cap \cdots \cap E_{i_{n}}\right)$ indicate the average intervalvalued intuitionistic fuzzy entropy provided by ex- 
perts $D_{i 1}, D_{i 2}, \cdots, D_{i n}$, where $E_{i_{1}} \cap E_{i_{2}} \cap \cdots \cap E_{i_{n}}$ is a real-valued entropy matrix.

Remark 1 This definition is established based on interval-valued intuitionistic fuzzy entropy. As we know, the entropy can be regarded as an important measure that reflects the decision making information to some extent. The smaller the entropy is, the greater the information will be. Therefore, consider the characteristics of the group decision making problems under interval-valued intuitionistic fuzzy environment, we utilize the minimize entropy principle to fuse the decision making information among the experts and establish the model above to obtain the experts absolute importance measure.

Due to the fact that importance measure should satisfy normalization condition, we should normalize the absolute measure before calculation. Hence, we define a new concept as follows:

Definition 10 Let $\mu\left(D_{i}\right)$ denote the relative importance measure of $D_{i}$, and $\overline{\mu\left(D_{i_{1}}, D_{i_{2}}, \cdots, D_{i_{n}}\right)}$ denotes the joint relative importance measure of $D_{i_{1}}, D_{i_{2}}, \cdots, D_{i_{n}}$, the experts relative importance measure is defined as follows:

$$
\overline{\mu\left(D_{i_{1}}, D_{i_{2}}, \cdots, D_{i_{n}}\right)}=\frac{\mu\left(D_{i_{1}}, D_{i_{2}}, \cdots, D_{i_{n}}\right)}{\mu\left(D_{1}, D_{2}, \cdots, D_{n}\right)}
$$

Theorem $5 \overline{\mu\left(D_{i_{1}}, D_{i_{2}}, \cdots, D_{i_{n}}\right)}$ satisfies the monotonicity of the fuzzy measure.

Proof. Since $\left\{D_{i_{1}}, D_{i_{2}}, \cdots, D_{i_{n}}\right\} \subseteq\left\{D_{i_{1}}, D_{i_{2}}, \cdots, D_{i_{n+1}}\right\}$, then

$$
\begin{aligned}
& \overline{\mu\left(D_{i_{1}}, D_{i_{2}}, \cdots, D_{i_{n}}, D_{i_{n+1}}\right)}-\overline{\mu\left(D_{i_{1}}, D_{i_{2}}, \cdots, D_{i_{n}}\right)} \\
& =\frac{\mu\left(D_{i_{1}}, D_{i_{2}}, \cdots, D_{i_{n}}, D_{i_{n+1}}\right)-\mu\left(D_{i_{1}}, D_{i_{2}}, \cdots, D_{i_{n}}\right)}{\mu\left(D_{1}, D_{2}, \cdots, D_{n}\right)} \\
& =\frac{1-E\left(E_{i_{1}} \cap E_{i_{2}} \cap \cdots \cap E_{i_{n}} \cap E_{i_{n+1}}\right)-\left(1-E\left(E_{i_{1}} \cap E_{i_{2}} \cap \cdots \cap E_{i_{n}}\right)\right)}{\mu\left(D_{1}, D_{2}, \cdots, D_{n}\right)} \\
& =\frac{E\left(E_{i_{1}} \cap E_{i_{2}} \cap \cdots \cap E_{i_{n}}\right)-E\left(E_{i_{1}} \cap E_{i_{2}} \cap \cdots \cap E_{i_{n}} \cap E_{i_{n+1}}\right)}{\mu\left(D_{1}, D_{2}, \cdots, D_{n}\right)} \geqslant 0
\end{aligned}
$$

So $\overline{\mu\left(D_{i_{1}}, D_{i_{2}}, \cdots, D_{i_{n}}\right)}$ satisfies the monotonicity of the fuzzy measure. The proof has been completed.

Remark 2 Theorem 5 indicates the fact that with the increasing number of experts (or decision makers), the information proposed by experts (or decision makers) is also increasing. It also states that the uncertainty of information reduces. Therefore, the experts relative importance measure proposed here is reasonable in real life decision making situations.

\subsection{Attributes importance measure based on weight information matrix}

Attribute importance measure is a key issue of the decision making problems in practice. As a useful tool to solve the attribute importance measure, Shannon entropy was widely used to trade off the importance among the attributes in real life decision making. Let $C_{i}$ denote the ith attribute, $H_{i}$ denote the Shannon entropy with respect to $C_{i}$. We usually measure the importance of $C_{i}$ based on the value of $H_{i}$. The smaller value of $H_{i}$, the great importance of $C_{i}$ is. In what follows, we define some new joint attributes information entropy based on original Shannon entropy to measure the importance of the attributes.

Definition 11 Let $H_{i}$ indicates the Shannon entropy with respect to the attribute $C_{i}$. Three types of joint attributes information entropy are defined as follows:

(1) $E \Delta_{1}\left(i_{n}\right)=\prod_{i=1}^{i_{n}}\left(1-H_{i}\right) \frac{\pi}{4}$;

(2) $E \Delta_{2}\left(i_{n}\right)=\sin \left(\prod_{i=i_{1}}^{i_{n}}\left(1-H_{i}\right) \frac{\pi}{4}\right)$;

(3) $E \Delta_{3}\left(i_{n}\right)=\tan \left(\prod_{i=i_{1}}^{i_{n}}\left(1-H_{i}\right) \frac{\pi}{4}\right)$.

where $I_{n}=\left\{i_{1}, i_{2}, \cdots, i_{n}\right\}$ is a index sequence such that $i_{n-1} \leqslant i_{n}, 1 \leqslant i_{n} \leqslant p$.

It is clear that $0 \leqslant E \Delta_{1}\left(i_{n}\right), E \Delta_{2}\left(i_{n}\right), E \Delta_{3}\left(i_{n}\right)<$ 1 , and $E \Delta_{1}\left(i_{n}\right), E \Delta_{2}\left(i_{n}\right), E \Delta_{3}\left(i_{n}\right)$ are all continuous, monotonic and decreasing functions of $I_{n}$. That is to say, if $\left|I_{n}\right|<\left|I_{n+1}\right|$ (where $\left|I_{n}\right|$ is the number of $\left.I_{n}\right)$, then $E \Delta_{j}\left(i_{n+1}\right)<E \Delta_{j}\left(i_{n}\right), j=1,2,3$, which means, with an increasing of the number of attributes, the uncertainty of the information is reduced. Therefore, we can use these three formulas to measure the importance of the attributes. Let $C_{I}=\left\{\left\{i_{1}, i_{2}, \cdots, i_{n}\right\} \mid i_{n} \geqslant i_{n-1}, 1 \leqslant i_{n} \leqslant p\right\}$ be a set of index sequence, then we derive the following Theorem 6. 
Theorem 6 For any $I_{n} \in C_{I}, E \Delta_{2}\left(I_{n}\right)<E \Delta_{1}\left(I_{n}\right)<$ $E \Delta_{3}\left(I_{n}\right)$.

Proof. Considering the sine function $y=\sin x$, we can clearly see that when $x \in\left(0, \frac{\pi}{2}\right)$,the sine function is a continuous, monotonic increasing with respect to $x$. Based on the theory of trigonometric function, we can easily infer the fact that if $x \in\left(0, \frac{\pi}{2}\right)$, then $\sin x<x<\tan x$. Let $x=\prod_{i=1}^{i_{n}}\left(1-H_{i}\right) \frac{\pi}{4}$, it is clear to see that $0<x=\prod_{i=1}^{i_{n}}\left(1-H_{i}\right) \frac{\pi}{4}<\frac{\pi}{4}<\frac{\pi}{2}$. Thus, we obtain the following inequalities:

$\sin \left(\prod_{i=1}^{i_{n}}\left(1-H_{i}\right) \frac{\pi}{4}\right)<\prod_{i=1}^{i_{n}}\left(1-H_{i}\right) \frac{\pi}{4}<\tan \left(\prod_{i=1}^{i_{n}}\left(1-H_{i}\right) \frac{\pi}{4}\right)$

Therefore, we have $E \Delta_{2}\left(i_{n}\right)<E \Delta_{1}\left(i_{n}\right)<E \Delta_{3}\left(i_{n}\right)$. The proof has been completed.

To determine the importance of attributes, $\mathrm{Xu}{ }^{41}$ proposed a useful weight solving method with incomplete information. A weight information matrix $W=\left(w_{i j}\right)_{m \times n}$ is established based on a multiobjective optimization model, whose elements $w_{i j}$ is the optimal weight solution corresponding to the alternatives. In what follows, we consider a multiattribute group decision-making problem with attributes interaction information, and give a formulation based on weight information matrix and the three types of joint attributes information entropy we proposed to determine the attributes important measure under interval-valued intuitionistic fuzzy environment.

Definition 12 Let $W=\left(w_{j i}\right)_{m \times n}$ be a weight information matrix, $w_{j i}$ indicates the optimal weight for the alternative $A_{j}$ with respect to the attribute $C_{i}$, $\mu\left(C_{i}\right)$ indicates the attribute importance measure with respect to the attribute $C_{i} . \mu\left(C_{i_{1}}, C_{i_{2}}, \cdots, C_{i_{n}}\right)$ indicates the attribute importance measure with respect to the attributes $C_{i_{1}}, C_{i_{2}}, \cdots, C_{i_{n}}$. Then the attributes importance measures are defined as:

$$
\begin{aligned}
& \mu\left(C_{i}\right) \\
& =\left(\begin{array}{l}
{\left[w_{i}^{1-\lambda}, w_{i}{ }^{(1-\lambda) E \Delta_{3}\left(i_{1}\right)}\right],} \\
{\left[\left(1-w_{i}{ }^{(1-\lambda) E \Delta_{2}\left(i_{1}\right)}\right)^{2}, 1-w_{i}{ }^{(1-\lambda) E \Delta_{1}\left(i_{1}\right)}\right]}
\end{array}\right)
\end{aligned}
$$

$$
\begin{aligned}
\mu\left(C_{i_{1}}, C_{i_{2}}, \cdots, C_{i_{n}}\right) & {\left[\left(\sum_{i=i_{1}}^{i_{n}} w_{i}\right)^{1-\lambda},\left(\sum_{i=i_{1}}^{i_{n}} w_{i}\right)^{(1-\lambda) E \Delta_{3}\left(i_{n}\right)}\right], } \\
= & {\left[\begin{array}{c}
\left(1-\left(\sum_{i=i_{1}}^{i_{n}} w_{i}\right)^{(1-\lambda) E \Delta_{2}\left(i_{n}\right)}\right)^{2}, \\
1-\left(\sum_{i=i_{1}}^{i_{n}} w_{i}\right)^{(1-\lambda) E \Delta_{1}\left(i_{n}\right)}
\end{array}\right] }
\end{aligned}
$$

where $\lambda$ indicates an interaction coefficient.

Theorem $7 \mu\left(C_{i_{1}}, C_{i_{2}}, \cdots, C_{i_{n}}\right)$ satisfies the monotonicity of the interval-valued intuitionistic fuzzy measure.

Proof. Based on the theory of inequality, since $-1 \leqslant \lambda \leqslant 1,0 \leqslant H \leqslant 1$, and $0<\sum_{i=i_{1}}^{i_{n}} w_{i} \leqslant 1$, so we have $0<\prod_{i=1}^{n}\left(1-H_{i}\right)<1,0<\prod_{i=1}^{n}\left(1-H_{i}\right) \frac{\pi}{4}<\frac{\pi}{4}$, and $0<\tan \left(\prod_{i=1}^{n}\left(1-H_{i}\right) \frac{\pi}{4}\right)<\tan \frac{\pi}{4}=1$. Since $\sum_{i=i_{1}}^{i_{n}} w_{i} \leqslant$ 1 and $(1-\lambda)>(1-\lambda) \tan \left(\prod_{i=i_{1}}^{i=i_{n}}\left(1-H_{i}\right) \frac{\pi}{4}\right)$, then $\left(\sum_{i=i_{1}}^{i_{n}} w_{i}\right)^{1-\lambda}<\left(\sum_{i=i_{1}}^{i_{n}} w_{i}\right)^{(1-\lambda) \tan \left(\prod_{i=1}^{n}\left(1-H_{i}\right) \frac{\pi}{4}\right)}$, $\left(1-\left(\sum_{i=i_{1}}^{i_{n}} w_{i}\right)^{(1-\lambda) \sin \left(\prod_{i=1}^{n}\left(1-H_{i}\right) \frac{\pi}{4}\right)}\right)^{2}<1-$ $\left(\sum_{i=i_{1}}^{i_{n}} w_{i}\right)^{(1-\lambda) \sin \left(\prod_{i=1}^{n}\left(1-H_{i}\right) \frac{\pi}{4}\right)}<1-\left(\sum_{i=i_{1}}^{i_{n}} w_{i}\right)^{(1-\lambda)\left(\prod_{i=1}^{n}\left(1-H_{i}\right) \frac{\pi}{4}\right)}$ $\left(\sum_{i=i_{1}}^{i_{n}} w_{i}\right)^{(1-\lambda) \tan \left(\prod_{i=1}^{n}\left(1-H_{i}\right) \frac{\pi}{4}\right)}+1-\left(\sum_{i=i_{1}}^{i_{n}} w_{i}\right)^{(1-\lambda) \prod_{i=1}^{n}\left(1-H_{i}\right) \frac{\pi}{4}}<$ $\left(\sum_{i=i_{1}}^{i_{n}} w_{i}\right)^{(1-\lambda) \prod_{i=1}^{n}\left(1-H_{i}\right) \frac{\pi}{4}}+1-\left(\sum_{i=i_{1}}^{i_{n}} w_{i}\right)^{(1-\lambda) \prod_{i=1}^{n}\left(1-H_{i}\right) \frac{\pi}{4}}=$

Then we prove the monotonicity of the intervalvalued intuitionistic fuzzy measure as follows:

(1) Let $\mu_{1}(i)=\left(\sum_{i=i_{1}}^{i_{n}} w_{i}\right)^{1-\lambda}$, then we have $\mu_{1}(i+$ 


$$
\begin{aligned}
& 1)-\mu_{1}(i)=\left(\sum_{i=i_{1}}^{i_{n+1}} w_{i}\right)^{1-\lambda}-\left(\sum_{i=i_{1}}^{i_{n}} w_{i}\right)^{1-\lambda}= \\
& e^{\ln \left(\sum_{i=i_{1}}^{i_{n+1}} w_{i}\right)^{1-\lambda}}-e^{\ln \left(\sum_{i=i_{1}}^{i_{n}} w_{i}\right)^{1-\lambda}}>0 .
\end{aligned}
$$

Thus, $\mu_{1}(i)$ is an increasing function with respect to $i$.

(2) Let $\mu_{2}(i)=\left(\sum_{i=i_{1}}^{i_{n}} w_{i}\right)^{(1-\lambda) \tan \left(\prod_{i=i_{1} i_{n}}\left(1-H_{i}\right) \frac{\pi}{4}\right)}$. Then

$$
\begin{aligned}
& \ln \mu_{2}(i+1)-\ln \mu_{2}(i) \\
& =(1-\lambda) \tan \left(\prod_{i=i_{1}}^{i_{n+1}}\left(1-H_{i}\right) \frac{\pi}{4}\right) \ln \left(\sum_{i=i_{1}}^{i_{n+1}} w_{i}\right) \\
& -(1-\lambda) \tan \left(\prod_{i=i_{1}}^{i_{n}}\left(1-H_{i}\right) \frac{\pi}{4}\right) \ln \left(\sum_{i=i_{1}}^{i_{n}} w_{i}\right) \\
& =(1-\lambda)\left(\tan \left(\prod_{i=i_{1}}^{i_{n+1}}\left(1-H_{i}\right) \frac{\pi}{4}\right) \ln \left(\sum_{i=i_{1}}^{i_{n+1}} w_{i}\right)\right. \\
& \left.-\tan \left(\prod_{i=i_{1}}^{i_{n}}\left(1-H_{i}\right) \frac{\pi}{4}\right) \ln \left(\sum_{i=i_{1}}^{i_{n}} w_{i}\right)\right) \\
& =(1-\lambda)\left(\ln \left(\sum_{i=i_{1}}^{i_{n+1}} w_{i}\right)^{\tan \left(\prod_{i=i_{1}}^{i_{n+1}}\left(1-H_{i}\right) \frac{\pi}{4}\right)}\right. \\
& \left.-\ln \left(\sum_{i=i_{1}}^{i_{n}} w_{i}\right)^{\tan \left(\prod_{i=i_{1}}^{i_{n}}\left(1-H_{i}\right) \frac{\pi}{4}\right)}\right) \\
& =(1-\lambda) \ln \frac{\left(\sum_{i=i_{1}}^{i_{n+1}} w_{i}\right){ }^{\tan \left(\prod_{i=i_{1}}^{i_{n+1}}\left(1-H_{i}\right) \frac{\pi}{4}\right)}}{\left(\sum_{i=i_{1}}^{i_{n}} w_{i}\right)}>0
\end{aligned}
$$

From $\ln \mu_{2}(i+1)-\ln \mu_{2}(i)>0 \Rightarrow \mu_{2}(i+1)>$ $\mu_{2}(i)$. So $\mu_{2}(i)$ is an increasing function with respect to $i$.
(3) Let $\mu_{3}(i)=\left(1-\left(\sum_{i=i_{1}}^{i_{n}} w_{i}\right)^{(1-\lambda) \sin \left(\prod_{i=1}^{n}\left(1-H_{i}\right) \frac{\pi}{4}\right)}\right)^{2}$

$$
\begin{aligned}
& \mu_{3}(i+1)-\mu_{3}(i) \\
& =\left(1-\left(\sum_{i=i_{1}}^{i_{n+1}} w_{i}\right)^{(1-\lambda) \sin \left(\prod_{i=i_{1}}^{i_{n+1}}\left(1-H_{i}\right) \frac{\pi}{4}\right)}\right)_{2}^{2} \\
& -\left(1-\left(\sum_{i=i_{1}}^{i_{n}} w_{i}\right)^{(1-\lambda) \sin \left(\prod_{i=i_{1}}^{i_{n}}\left(1-H_{i}\right) \frac{\pi}{4}\right)}\right)^{2} \\
& =\left(2-\left(\sum_{i=i_{1}}^{i_{n+1}} w_{i}\right)^{(1-\lambda) \sin \left(\prod_{i=i_{1}}^{i_{n+1}}\left(1-H_{i}\right) \frac{\pi}{4}\right)}\right. \\
& \left.-\left(\sum_{i=i_{1}}^{i_{n}} w_{i}\right)^{(1-\lambda) \sin \left(\prod_{i=i_{1}}^{i_{n+1}}\left(1-H_{i}\right) \frac{\pi}{4}\right)}\right) \\
& \times\left(\left(\sum_{i=i_{1}}^{i_{n}} w_{i}\right)^{(1-\lambda) \sin \left(\prod_{i=i_{1}}^{i_{n}}\left(1-H_{i}\right) \frac{\pi}{4}\right)}\right. \\
& \left.-\left(\sum_{i=i_{1}}^{i_{n+1}} w_{i}\right)^{(1-\lambda) \sin \left(\prod_{i=i_{1}}^{i_{n+1}}\left(1-H_{i}\right) \frac{\pi}{4}\right)}\right)<0
\end{aligned}
$$

From $\mu_{3}(i+1)-\mu_{3}(i)<0$, we can obtain $\mu_{3}(i+$ $1)<\mu_{3}(i)$. Hence, $\mu_{3}(i)$ is a decreasing function with respect to $i$.

(4) The proof of (4) is similar to the proofs of (1)(3), omitted.

Hence, we verify the proposed fuzzy measure satisfies the properties of the interval-valued intuitionistic fuzzy sets and when $i_{1}=i_{2}=\cdots=i_{n}$, Eq. (23) is reduced to Eq. (22). Thus, the proof is complete.

In this section, we give a new method to solve experts importance measure and attributes importance measure under interval-valued intuitionistic fuzzy environment. Since the given approach based on interval-valued intuitionistic fuzzy Choquet integral, which greatly reduces the complexity of determin- 
ing the fuzzy measures, and improves the practicality of the method.

\section{An approach to multi-attribute group decision-making based on interval-valued intuitionistic fuzzy Choquet integral}

Interval-valued intuitionistic fuzzy multiple attribute group decision making task is a special case of fuzzy multiple attribute group decision making problems. In the sequel, based on the interval-valued intuitionistic fuzzy entropy and the interval-valued intuitionistic fuzzy measure we derived above, a new approach is proposed to solve multiple attribute group decision making problem under interval-valued intuitionistic fuzzy environment. We propose two methods to determine the fuzzy measure which are used to calculate the interactive importance measure of attributes and its correlation measure of experts (e.g. absolute measure, relative measure). Firstly, we describe the interval-valued intuitionistic fuzzy multiple attribute group decision making (MAGDM) problems in this paper.

\subsection{The description of MAGDM problems}

For an interval-valued intuitionistic fuzzy multiple attribute group decision making problem. Let $A=\left\{A_{1}, A_{2}, \cdots, A_{m}\right\}$ be a discreet set of alternatives, $C=\left\{C_{1}, C_{2}, \cdots, C_{n}\right\}$ be a set of attributes, and $D=\left\{D_{1}, D_{2}, \cdots, D_{p}\right\}$ be a set of experts. The performance of the alternative $A_{i}$ with respect to the attribute $C_{j}$ which is provided by expert $D_{k}$ is expressed by an IVIFN $A_{i j}^{(k)}=$ $\left(\left[a_{i j k}^{L}, a_{i j k}^{R}\right],\left[b_{i j k}^{L}, b_{i j k}^{R}\right]\right)$, where $\left[a_{i j k}^{L}, a_{i j k}^{R}\right]$ indicates the interval-valued degrees that the alternative $A_{i}$ satisfies the attribute $C_{j}$, and $\left[b_{i j k}^{L}, b_{i j k}^{R}\right]$ indicates the interval-valued degrees that the alternative $A_{i}$ does not satisfies the attribute $C_{j}$, such that $\left[a_{i j k}^{L}, a_{i j k}^{R}\right] \subseteq[0,1],\left[b_{i j k}^{L}, b_{i j k}^{R}\right] \subseteq[0,1]$ and $0 \leqslant$ $a_{i j k}^{R}+b_{i j k}^{R} \leqslant 1$. For all elements $A_{i j}^{(k)}$ are contained in the decision matrix $D M_{(k)}$ which is provided by expert $D_{k}$. Based on the analysis presented before, we develop a new method for MAGDM problems under interval-valued intuitionistic fuzzy environment.
Based on these necessary conditions, the ranking of alternatives is required.

\subsection{Decision making steps}

The interval-valued intuitionistic fuzzy Choquet integral method is illustrated in what follows. The algorithm involves the following steps:

Step 1 Construct the individual decision matrix $D M_{(k)}(k=1,2, \cdots, p)$.

Step 2 Calculate the entropy of each decision matrix $E_{k}(k=1,2, \cdots, p)$.

Step 3 Calculate the importance measure based on the entropy of experts in accordance with decision matrix.

(1) Calculate the absolute measure

With the use of Definition 9 and Eqs. (17-19), we obtain the experts absolute importance measure.

(2) Calculate the relative measure

Using Definition 10 and Eq. (20), we determine the experts relative importance measure.

Step 4 Calculate the weights of experts.

Based on game theory, we get the expert weights with Shapely value ${ }^{60}$.

$$
\sigma_{k}(v)=\frac{(p-|k|) !(|k|-1) !}{p !}\left(\overline{\mu\left(D_{S}\right)}-\overline{\mu\left(D_{S-\{i\}}\right)}\right)
$$

where $\sigma_{k}(v)$ indicates the expert weight of $D_{k}$.

Step 5 Aggregate the decision information of each expert to collect into group decision matrix $G$ by using the IVIFWA operator.

Utilize the IVIFWA operator to arrange the individual decision matrices into a collective (aggregate) decision matrix as follows:

$$
G=\left(\left[g_{\mu_{i j}}^{L}, g_{\mu_{i j}}^{L}\right],\left[g_{v_{i j}}^{L}, g_{v_{i j}}^{L}\right]\right)_{m \times n}
$$

Step 6 Calculate the group decision score matrix and the entropy matrix, respectively.

With Eqs. (2) and (3), we calculate the score matrix $S_{G}$ and entropy matrix $E_{G}$ respectively, which 
are defined as follows:

$$
\begin{aligned}
S_{G} & =\left[\begin{array}{cccc}
s_{11} & s_{12} & \cdots & s_{1 n} \\
s_{21} & s_{22} & \cdots & s_{2 n} \\
\cdots & \cdots & \cdots & \cdots \\
s_{m 1} & s_{m 2} & \cdots & s_{m n}
\end{array}\right] \\
E_{G} & =\left[\begin{array}{cccc}
E_{11} & E_{12} & \cdots & E_{1 n} \\
E_{21} & E_{22} & \cdots & E_{2 n} \\
\cdots & \cdots & \cdots & \cdots \\
E_{m 1} & E_{m 2} & \cdots & E_{m n}
\end{array}\right]
\end{aligned}
$$

Step 7 Construct the programming model of attribute weights based on the relative entropy measure.

Based on the definition of relative entropy, and with the purpose to determine the optimal weight vector, we have the basic idea to maximize the score function of each alternative, and minimize the entropy function of each alternative. On the basis of the above analysis, we establish the following linear programming model corresponding to the $i$-th alternative:

$$
\begin{aligned}
& \min W_{i}=\sum_{j=1}^{n} w_{i j} \ln \left|\frac{E_{i j}}{s_{i j}}\right|, i=1,2, \cdots m \\
& \text { s.t. }\left\{\begin{array}{l}
\sum_{j=1}^{n} w_{i j}=1 \\
w_{i j} \geqslant 0
\end{array}\right.
\end{aligned}
$$

By solving the above sated linear programming model, we produce the solution of the attribute weight vectors $W^{(i)}=\left(w_{1}^{(i)}, w_{2}^{(i)}, \cdots w_{n}^{(i)}\right)$, where $i=$ $1,2, \cdots, m$.

Step 8 Solve the optimal attribute weights vector.

Based on the definition of Shannon entropy, we can determine the attribute weight vector as follows:

$$
\begin{gathered}
H_{j}=-\frac{1}{m \ln 2} \sum_{i=1}^{m} w_{i j}^{(i)} \ln w_{i j}^{(i)}(j=1,2, \cdots, n) \\
w_{j}=\frac{1-H_{j}}{n-\sum_{k=1}^{n} H_{k}}(j=1,2, \cdots, n)
\end{gathered}
$$

Step 9 Calculate the interaction coefficient $\lambda$.

In virtue of Eq. (5), calculate the value of parameter $\lambda$ by solving the following non-linear multiobjective programming problem:
According to the principle of optimization, we can construct a single-objective programming model to solve this multiple-objective programming:

$$
\begin{aligned}
& \min \frac{1}{2}\left[\left(\prod_{i=1}^{n}\left(1+\lambda H_{i}\right)-(\lambda+1)\right)^{2}-\sum_{i=1}^{n} H_{i}+\right. \\
& \left.\sum_{i=1}^{n} \frac{1}{\lambda}\left(\prod_{i=1}^{n}\left[1+\lambda H_{i}\right]-1\right) \ln \left(\frac{1}{\lambda}\left(\prod_{i=1}^{n}\left[1+\lambda H_{i}\right]-1\right)\right)\right] \\
& \text { s.t. }\left\{\begin{array}{l}
-1 \leqslant \lambda \leqslant 1 \\
\lambda \neq 0
\end{array}\right.
\end{aligned}
$$

Step 10 Calculate the importance measure of attribute weights.

Based on Eqs. (22-23), form the fuzzy measure of attribute weights.

Step 11 Utilize the Choquet integral to aggregate the interval-valued intuitionistic fuzzy information of decision-making matrix.

Based on Definition 8 and Eq. (16), the aggregate value of the alternatives is formed.

Step 12 Use the score function of IVIFS to obtain the score of overall alternatives.

Based on Eq. (2), we form the score value of the alternatives.

Step 13 Utilize the score value to rank the alternatives in the descending order.

Rank all the alternatives by Eq. (2), the larger the score value is, the better the alternative is.

Step 14 End.

\section{Numerical example}

In this section, we provide an example to show the application of the proposed method for supplier selection problem.

\subsection{The supplier selection problem description}

With the continuous development of economic globalization, the supply chain management has played an important role in marketing economic and become the most hot research topic in modern management science, which directly impact on the manu- 
factures performance. Consider a problem in a shipbuilding company, which aims to search for the best supplier for purchasing ship equipments. Three potential ship equipments suppliers have been identified. The attributes to be considered in the evaluation process are: $C_{1}$ : business management capacity; $C_{2}$ : quality; $C_{3}$ : transportation and delivery ability; $C_{4}$ : service ability. The three shipbuilding companies are to be evaluated using the intervalvalued intuitionistic fuzzy information by three experts $D_{k}(k=1,2,3)$.

\subsection{Illustration of the proposed method}

Step 1 Construct the individual decision matrix $\operatorname{DM}_{(k)}(k=1,2,3)$, respectively.

$$
\begin{aligned}
& D M_{(1)}=\left(\begin{array}{cc}
([0.3,0.4],[0.4,0.6]) & ([0.5,0.6],[0.1,0.2]) \\
([0.6,0.8],[0.1,0.2]) & ([0.6,0.7],[0.2,0.3]) \\
([0.5,0.8],[0.1,0.2]) & ([0.7,0.8],[0.0,0.2]) \\
([0.6,0.7],[0.2,0.3]) & ([0.7,0.8],[0.0,0.1]) \\
([0.2,0.3],[0.4,0.6]) & ([0.5,0.6],[0.1,0.3]) \\
([0.5,0.5],[0.4,0.5]) & ([0.2,0.3],[0.2,0.4])
\end{array}\right) \\
& D M_{(2)}=\left(\begin{array}{cc}
([0.4,0.5],[0.3,0.4]) & ([0.5,0.6],[0.1,0.2]) \\
([0.6,0.8],[0.1,0.2]) & ([0.5,0.6],[0.3,0.4]) \\
([0.5,0.6],[0.3,0.4]) & ([0.5,0.7],[0.1,0.2]) \\
([0.6,0.7],[0.2,0.3]) & ([0.7,0.8],[0.1,0.2]) \\
([0.4,0.5],[0.3,0.4]) & ([0.4,0.6],[0.3,0.4]) \\
([0.5,0.6],[0.3,0.4]) & ([0.3,0.4],[0.2,0.5])
\end{array}\right) \\
& D M_{(3)}=\left(\begin{array}{cc}
([0.4,0.6],[0.3,0.4]) & ([0.5,0.7],[0.0,0.2]) \\
([0.5,0.8],[0.1,0.2]) & ([0.3,0.5],[0.2,0.3]) \\
([0.5,0.6],[0.0,0.1]) & ([0.5,0.8],[0.1,0.2]) \\
([0.5,0.6],[0.2,0.4]) & ([0.6,0.8],[0.1,0.2]) \\
([0.3,0.6],[0.2,0.4]) & ([0.4,0.5],[0.2,0.4]) \\
([0.4,0.7],[0.2,0.3]) & ([0.2,0.4],[0.2,0.3])
\end{array}\right)
\end{aligned}
$$

Step 2 Calculate the entropy of each individual decision matrix $E_{k}(k=1,2,3)$, respectively.

$$
\begin{aligned}
& E_{1}=\left(\begin{array}{llll}
0.9791 & 0.8855 & 0.8412 & 0.6087 \\
0.7308 & 0.8413 & 0.9510 & 0.9048 \\
0.7705 & 0.6310 & 0.9972 & 0.9985
\end{array}\right) \\
& E_{2}=\left(\begin{array}{llll}
0.9914 & 0.8854 & 0.8412 & 0.6548 \\
0.7308 & 0.9594 & 0.9914 & 0.9790 \\
0.9594 & 0.8443 & 0.9594 & 0.9927
\end{array}\right) \\
& E_{3}=\left(\begin{array}{llll}
0.9791 & 0.8229 & 0.9422 & 0.7308 \\
0.7933 & 0.9848 & 0.9823 & 0.9822 \\
0.8467 & 0.7933 & 0.9238 & 0.9985
\end{array}\right)
\end{aligned}
$$

Step 3 Calculate the importance measure based on the entropy of expert in accordance with the obtained decision matrix.

Based on Defintion 10 and Eqs.(11-13), we calculate the experts importance measure. The obtained results are shown as follows:

$\mu\left(D_{1}\right)=0.1551, \mu\left(D_{2}\right)=0.1009, \mu\left(D_{3}\right)=0.1017$

$$
\begin{aligned}
& \mu\left(D_{1}, D_{2}\right) \\
& =1-E\left[\begin{array}{llll}
0.9791 & 0.8854 & 0.8412 & 0.6087 \\
0.7308 & 0.8413 & 0.9510 & 0.9048 \\
0.7705 & 0.6310 & 0.9594 & 0.9927
\end{array}\right] \\
& =1-0.8413=0.1587
\end{aligned}
$$

Similarly, we form other fuzzy measures based on the entropy of interval-valued intuitionistic fuzzy information. The results are calculated as follows:

$$
\begin{aligned}
& \mu\left(D_{1}, D_{3}\right)=0.1664, \mu\left(D_{2}, D_{3}\right)=0.1245, \\
& \mu\left(D_{1}, D_{2}, D_{3}\right)=0.1669
\end{aligned}
$$

Then we use Eq. (20) to normalize the absolute measure, and obtain the experts importance measures:

$$
\begin{aligned}
& \overline{\mu\left(D_{1}\right)}=0.9292, \overline{\mu\left(D_{2}\right)}=0.6045, \overline{\mu\left(D_{3}\right)}=0.6093, \\
& \overline{\mu\left(D_{1}, D_{2}\right)}=0.9508, \overline{\mu\left(D_{1}, D_{3}\right)}=0.9970, \\
& \overline{\mu\left(D_{2}, D_{3}\right)}=0.7459, \overline{\mu\left(D_{1}, D_{2}, D_{3}\right)}=1
\end{aligned}
$$

Step 4 Calculate the weight of experts.

According to the results of game theory, we get the expert weight based on Shapely-value. The re- 
sults read shown as follows:

$$
\begin{aligned}
\sigma_{1}(v) & =\frac{(3-1) !(1-1) !}{3 !} \overline{\mu\left(D_{1}\right)} \\
& +\frac{(3-2) !(2-1) !}{3 !}\left(\overline{\mu\left(D_{1}, D_{2}\right)}-\overline{\mu\left(D_{2}\right)}\right) \\
& \left.+\frac{(3-2) !(2-1) !}{3 !} \overline{\mu\left(D_{1}, D_{3}\right)}-\overline{\mu\left(D_{3}\right)}\right) \\
& \left.+\frac{(3-3) !(3-1) !}{3 !} \overline{\mu\left(D_{1}, D_{2}, D_{3}\right)}-\overline{\mu\left(D_{2}, D_{3}\right)}\right) \\
& =\frac{1}{3} \times 0.9292+\frac{1}{6} \times(0.9508-0.6045) \\
& +\frac{1}{6} \times(0.9970-0.6093)+\frac{1}{3} \times(1-0.7459) \\
& =0.4451 \\
\sigma_{2}(v) & =\frac{1}{3} \times 0.6045+\frac{1}{6} \times(0.9508-0.9292) \\
& +\frac{1}{6} \times(0.7459-0.6093)+\frac{1}{3} \times(1-0.9970) \\
& =0.2288 \\
\sigma_{3}(v) & =\frac{1}{3} \times 0.6093+\frac{1}{6} \times(0.9970-0.9292) \\
& +\frac{1}{6} \times(0.7459-0.6045)+\frac{1}{3} \times(1-0.9508) \\
& =0.2543
\end{aligned}
$$

Normalize the results of $\sigma_{i}(v)(i=1,2,3)$, which leads to the weight information corresponding to the three experts:

$$
\begin{aligned}
& w_{D_{1}}=\frac{\sigma_{1}(v)}{\sigma_{1}(v)+\sigma_{2}(v)+\sigma_{3}(v)}=0.4795 \\
& w_{D_{2}}=\frac{\sigma_{2}(v)}{\sigma_{1}(v)+\sigma_{2}(v)+\sigma_{3}(v)}=0.2465 \\
& w_{D_{3}}=\frac{\sigma_{3}(v)}{\sigma_{1}(v)+\sigma_{2}(v)+\sigma_{3}(v)}=0.2740
\end{aligned}
$$

Step 5 Aggregate the decision information of each expert to collect a group decision matrix $G$ by using the IVIFWA operator.

Utilize the IVIFWA operator, and then structure the decision information in the form of the group de- cision matrix.

$$
\begin{aligned}
& G= \\
& {\left[\begin{array}{ll}
([0.35,0.48],[0.34,0.48]) & ([0.50,0.63],[0.00,0.20]) \\
([0.57,0.80],[0.10,0.20]) & ([0.51,0.63],[0.22,0.32]) \\
([0.50,0.71],[0.00,0.19]) & ([0.61,0.77],[0.00,0.20]) \\
([0.57,0.68],[0.20,0.32]) & ([0.67,0.80],[0.00,0.14]) \\
([0.36,0.44],[0.31,0.48]) & ([0.45,0.57],[0.16,0.35]) \\
([0.47,0.59],[0.30,0.41]) & ([0.25,0.35],[0.20,0.39])
\end{array}\right]}
\end{aligned}
$$

Step 6 Calculate the group decision score matrix and the entropy matrix, respectively.

Based on the Definitions 3 and 4, the score matrix $S_{G}$ and entropy matrix $E_{G}$ come in the form:

$$
\begin{aligned}
& S_{G}=\left[\begin{array}{llll}
0.005 & 0.465 & 0.365 & 0.665 \\
0.535 & 0.300 & 0.005 & 0.255 \\
0.510 & 0.590 & 0.175 & 0.005
\end{array}\right] \\
& E_{G}=\left[\begin{array}{llll}
0.999 & 0.854 & 0.871 & 0.642 \\
0.751 & 0.918 & 0.998 & 0.947 \\
0.816 & 0.721 & 0.969 & 0.998
\end{array}\right]
\end{aligned}
$$

Step 7 Construct the programming model of attribute weights based on relative entropy.

The information about attribute weights given by the decision makers can be described as follows:

- $\Omega_{D_{1}}: 0.1 \leqslant w_{1} \leqslant 0.3, w_{2}-w_{1} \geqslant 0.15$

- $\Omega_{D_{2}}: 0.1 \leqslant w_{2} \leqslant 0.4,0.2 \leqslant w_{3} \leqslant 0.3$

- $\Omega_{D_{3}}: w_{3} \leqslant w_{4}, w_{4}-w_{2} \leqslant w_{3}-w_{1}$

Then we establish a linear programming problem:

$$
\begin{array}{r}
\min W_{i}=5.29 w_{1}+6.07 w_{2}+0.87 w_{3}-0.035 w_{4} \\
\text { s.t. }\left\{\begin{array}{l}
w_{1}+w_{2}+w_{3}+w_{4}=1 \\
w_{i} \geqslant 0, \Omega_{D_{k}}, k=1,2,3
\end{array}\right.
\end{array}
$$

By solving this problem, we can get the optimal weight vector is $W^{(1)}=$ $(0.10,0.25,0.25,0.40)$. Similarly, we get $W^{(2)}=$ $(0.23,0.37,0.20,0.20), W^{(3)}=(0.20,0.40,0.20,0.20)$. Hence, the optimal weight information matrix becomes:

$$
W=\left[\begin{array}{llll}
0.10 & 0.25 & 0.25 & 0.40 \\
0.23 & 0.37 & 0.20 & 0.20 \\
0.20 & 0.40 & 0.20 & 0.20
\end{array}\right]
$$

Step 8 Solve the optimal attribute weights vector.

Based on Eq. (28), we derive the value of $H_{i}(i=$ 1,2,3,4) as follows: $H_{1}=0.428, H_{2}=0.265, H_{3}=$ 
$0.399, H_{4}=0.376$. Utilize Eq. (29) to calculate the weights of attributes:

$$
\begin{aligned}
& w_{1}=\frac{1-H_{1}}{4-\sum_{k=1}^{3} H_{k}}=\frac{1-0.428}{4-1.468}=0.2259, \\
& w_{2}=\frac{1-H_{2}}{4-\sum_{k=1}^{3} H_{k}}=\frac{1-0.265}{4-1.468}=0.2902, \\
& w_{3}=\frac{1-H_{3}}{4-\sum_{k=1}^{3} H_{k}}=\frac{1-0.399}{4-1.468}=0.2373, \\
& w_{4}=4 \frac{1-H_{4}}{3-\sum_{k=1}^{3} H_{k}}=\frac{1-0.376}{4-1.468}=0.2466 .
\end{aligned}
$$

Step 9 Calculate the interaction coefficient $\lambda$.

Based on Eq. (5), we calculate the interaction coefficient to be $\lambda=-0.6925$.

Step 10 Establish the importance measure of attribute weights.

Based on Eqs. (22-23), we obtain the fuzzy measures directly. The results are shown as follows:

$$
\begin{aligned}
\mu\left(C_{1}\right) & =([0.0806,0.2800],[0.4318,0.6773]), \\
\mu\left(C_{2}\right) & =([0.1232,0.2261],[0.4463,0.7014]), \\
\mu\left(C_{3}\right) & =([0.0876,0.2696],[0.4367,0.6831]), \\
\mu\left(C_{4}\right) & =([0.0935,0.2619],[0.4394,0.6869]), \\
\mu\left(C_{1}, C_{2}\right) & =([0.3264,0.6813],[0.0927,0.3090]), \\
\mu\left(C_{1}, C_{3}\right) & =([0.2718,0.6973],[0.0861,0.2965]), \\
\mu\left(C_{1}, C_{4}\right) & =([0.2811,0.6940],[0.0877,0.2993]), \\
\mu\left(C_{2}, C_{3}\right) & =([0.3387,0.6761],[0.0948,0.3131]), \\
\mu\left(C_{2}, C_{4}\right) & =([0.3489,0.6726],[0.0961,0.3157]), \\
\mu\left(C_{3}, C_{4}\right) & =([0.2927,0.6889],[0.0900,0.3036]), \\
\mu\left(C_{1}, C_{2}, C_{3}\right) & =([0.6192,0.9081],[0.0127,0.0907]), \\
\mu\left(C_{1}, C_{2}, C_{4}\right) & =([0.6322,0.9086],[0.0126,0.0901]), \\
\mu\left(C_{1}, C_{3}, C_{4}\right) & =([0.7098,0.8143],[0.0714,0.1855]), \\
\mu\left(C_{2}, C_{3}, C_{4}\right) & =([0.6483,0.9091],[0.0124,0.0896]), \\
\mu\left(C_{1}, C_{2}, C_{3}, C_{4}\right) & =([1,1],[0,0]) .
\end{aligned}
$$

Step 11 Utilize Choquet integral to aggregate the interval-valued intuitionistic fuzzy information of decision matrix.
Take $A_{1}$ as an example. From Definition 8 and Eq. (16), we have:

$$
\begin{aligned}
\mu_{A_{1}}^{L} & =0.35 \times\left(\mu(C)-\mu\left(C_{2}, C_{3}, C_{4}\right)\right) \\
& +0.50 \times\left(\mu\left(C_{2}, C_{3}, C_{4}\right)-\mu\left(C_{3}, C_{4}\right)\right) \\
& +0.57 \times\left(\mu\left(C_{3}, C_{4}\right)-\mu\left(C_{4}\right)\right) \\
& +0.67 \times\left(\mu\left(C_{4}\right)-\mu(\phi)\right) \\
& =0.35 \times(1-0.6483)+0.50 \times(0.6483-0.2927) \\
& +0.57 \times(0.2927-0.0935)+0.67 \times(0.0935-0) \\
& =0.4771 \\
\mu_{A_{1}}^{R} & =0.48 \times\left(\mu(C)-\mu\left(C_{2}, C_{3}, C_{4}\right)\right) \\
& +0.63 \times\left(\mu\left(C_{2}, C_{3}, C_{4}\right)-\mu\left(C_{3}, C_{4}\right)\right) \\
& +0.68 \times\left(\mu\left(C_{3}, C_{4}\right)-\mu\left(C_{4}\right)\right) \\
& +0.80 \times\left(\mu\left(C_{4}\right)-\mu(\phi)\right) \\
& =0.48 \times(1-0.9091)+0.63 \times(0.9091-0.6889) \\
& +0.68 \times(0.6889-0.2619)+0.80 \times(0.2619-0) \\
& =0.6843 \\
v_{A_{1}}^{L} & =0.34 \times\left(\mu\left(C_{1}, C_{2}, C_{4}\right)-\mu(C)\right) \\
& +0.2 \times\left(\mu\left(C_{2}, C_{4}\right)-\mu\left(C_{1}, C_{2}, C_{4}\right)\right) \\
& +0 \times\left(\mu\left(C_{4}\right)-\mu\left(C_{2}, C_{4}\right)\right) \\
& +0 \times\left(\mu(\phi)-\mu\left(C_{4}\right)\right) \\
& =0.34 \times(0.0126-0)+0.2 \times(0.0961-0.0126) \\
& +0 \times(0.4394-0.0961)+0 \times(1-0.4394) \\
& =0.0210 \\
v_{A_{1}}^{R} & =0.48 \times\left(\mu\left(C_{2}, C_{3}, C_{4}\right)-\mu(C)\right) \\
& +0.32 \times\left(\mu\left(C_{2}, C_{4}\right)-\mu\left(C_{2}, C_{3}, C_{4}\right)\right) \\
& +0.2 \times\left(\mu\left(C_{4}\right)-\mu\left(C_{2}, C_{4}\right)\right) \\
& +0.14 \times\left(\mu(\phi)-\mu\left(C_{4}\right)\right) \\
& =0.48 \times(0.0896-0)+0.32 \times(0.3157-0.0896) \\
& +0.2 \times(0.6869-0.3157)+0.14 \times(1-0.6869) \\
& =0.2334 \\
&
\end{aligned}
$$

So the aggregation value of $A_{1}$ based on intervalvalued intuitionistic fuzzy Choquet integral is:

(c) $A_{1}=([0.4771,0.6843],[0.0210,0.2334])$ 
Similarly, we can calculate the aggregation values of $A_{2}$ and $A_{3}$, respectively:

$$
\begin{aligned}
& \text { (c) } A_{2}=([0.4738,0.6607],[0.2652,0.3055]) \\
& (c) A_{3}=([0.4631,0.6524],[0.2266,0.3287])
\end{aligned}
$$

Step 12 Utilize the score function to get the score value of the overall alternatives.

Based on Eq. (2), we can calculate the score value of overall alternatives, respectively:

$$
S\left(A_{1}\right)=0.4518, S\left(A_{2}\right)=0.2819, S\left(A_{3}\right)=0.2801
$$

Step 13 Utilize the score value to rank the alternatives.

Since $S\left(A_{1}\right)>S\left(A_{2}\right)>S\left(A_{3}\right)$, we have $A_{1} \succ$ $A_{2} \succ A_{3}$. Thus, we choose $A_{1}$ as the best alternative.

\subsection{Comparisons and further discussion}

In what follows, we compare our method with other existing methods including interval-valued intuitionistic fuzzy group decision making with Choquet integral-based TOPSIS ${ }^{42}$, and the intervalvalued intuitionistic fuzzy Choquet integral with respect to the generalized lambda-Shapley index method ${ }^{50}$. The results are shown in Table 1 .

Table 1. Comparasions with Choquet integral- TOPSIS and generalized lambda-Shapley index methods.

\begin{tabular}{lc}
\hline Methods & Order of alternatives \\
\hline The proposed method & $A_{1} \succ A_{2} \succ A_{3}$ \\
Choquet integral-based TOPSIS & $A_{1} \succ A_{2} \succ A_{3}$ \\
lambda-Shapley index method & $A_{1} \succ A_{2} \succ A_{3}$ \\
\hline
\end{tabular}

From Table 1, it is clear to see that three methods have the same ranking results. This verifies the method we proposed is reasonable and validity in this study.

(1) Compared with the Choquet integral-based TOPSIS which was proposed by Tan ${ }^{42}$. The main advantage of our method is that we develop a new method to determine the fuzzy measures directly based on decision information, while in Tan's method, the fuzzy measures need provided by the decision maker which maybe difficult to the DMs and is closely related the subjective preferences of the DMs, or even lead to inconsistent results. In addition, our method makes full use of weights information to characterize the interrelationships among the attributes, which ensures the ranking results are more objective and accurate.

(2) Compared with the generalized lambda-Shapley index method which was proposed by Meng et al. ${ }^{50}$. The computational complexity of our method is much lower than Mengs method. The reason is that our method is a constructive method, which only needs to determine a single parameter by solving a simple linear programming model, and then the fuzzy measures can be easily obtained, whereas Meng's method needs to solve complex programming model, which leads to high computational complexity and information loss. Moreover, our method can solve the MAGDM with incomplete known information, while generalized lambda-Shapley index method does not deal with this problem. Therefore, our method is more general.

According to the comparisons and analysis above, the method we proposed is better than the other two methods. Therefore, our method is more suitable to solve interval-valued intuitionistic fuzzy multiple attribute group decision making.

\section{Conclusions}

In this study, we have presented a new approach to determine the fuzzy measure of Choquet integral on the basis of interval-valued intuitionistic fuzzy decision matrix. Considering the interval-valued intuitionistic fuzzy entropy theory, we give a simple solution method to determine the interval-valued intuitionistic fuzzy measures and present a method to obtain the experts weights and attributes weights with fuzzy measures directly. The strict mathematical reasoning shows that our new proposed method satisfies the axioms of interval-valued intuitionistic fuzzy measures. Then, the experts weights and attributes weights are aggregated by using the Shapely values. The prominent advantage of such fuzzy 
measure method is that it can ease the burden of the decision maker, avoid the information losing and distortion and improve the accuracy of the decision making results. Based on our theoretical analysis results, we develop a new Choquet integral-based approach to solve the interval-valued intuitionistic fuzzy multiple attribute decision making problems. The fuzzy measures to determine the attribute weights and experts weights are generated by relative entropy models. A solution process is proposed to solve the intervalCvalued intuitionistic fuzzy multiple attribute group decision making problems. Our new method is simple prerequisite conditions and can be easily integrated into other multi-attribute group decision-making techniques, which are effective expansions on the current interval-valued intuitionistic fuzzy decision making models and solution methods. Furthermore, our new methods can also be applied to the similar problems in the forms of linguistic variables hesitate fuzzy sets and type-2 fuzzy sets. These will be considered in the future research.

\section{Acknowledgments}

The work is supported by the National Natural Science Foundation of China (NSFC) under Projects 71171048 and 71371049, Ph.D. Program Foundation of Chinese Ministry of Education 20120092110038, the Scientific Research and Innovation Project for College Graduates of Jiangsu Province CXZZ13_0138, and the Scientific Research Foundation of Graduate School of Southeast University YBJJ1454.

\section{References}

1. L.A. Zadeh, Fuzzy sets, Information and control, $\mathbf{8}$ (1965) 338-353.

2. D.-C. Lin, J.-S. Yao, Fuzzy economic production for production inventory, Fuzzy sets and systems, 111 (2000) 465-495.

3. Z. Hu, C. Rao, Y. Zheng, D. Huang, Optimization Decision of Supplier Selection in Green Procurement under the Mode of Low Carbon Economy, International Journal of Computational Intelligence Systems, 8 (2015) 407-421.

4. X. Huang, Mean-entropy models for fuzzy portfolio selection, IEEE Transactions on Fuzzy Systems, 16
(2008) 1096-1101.

5. A. Bonetti, S. Bortot, M. Fedrizzi, R.M. Pereira, A. Molinari, Modelling group processes and effort estimation in project management using the Choquet integral: An MCDM approach, Expert Systems with Applications, 39 (2012) 13366-13375.

6. I. Saad, S. Hammadi, M. Benrejeb, P. Borne, Choquet integral for criteria aggregation in the flexible jobshop scheduling problems, Mathematics and Computers in Simulation, 76 (2008) 447-462.

7. D. Wu, G. Zhang, J. Lu, A fuzzy preference treebased recommender system for personalized businessto-business e-services, IEEE Transactions on Fuzzy Systems, 23 (2015) 29-43.

8. S. Han, J.M. Mendel, A new method for managing the uncertainties in evaluating multi-person multi-criteria location choices, using a perceptual computer, Annals of Operations Research, 195 (2012) 277-309.

9. W. Pedrycz, From fuzzy data analysis and fuzzy regression to granular fuzzy data analysis, Fuzzy Sets and Systems, 274 (2015) 12-17.

10. S. Srivastava, M. Singh, V.K. Madasu, M. Hanmandlu, Choquet fuzzy integral based modeling of nonlinear system, Applied Soft Computing, 8 (2008) 839-848.

11. S. Dhompongsa, A. Kaewkhao, S. Saejung, On topological properties of the Choquet weak convergence of capacity functionals of random sets, Information Sciences, 177 (2007) 1852-1859.

12. S. Wang, W. Pedrycz, Q. Zhu, W. Zhu, Subspace learning for unsupervised feature selection via matrix factorization, Pattern Recognition, 48 (2015) 10-19.

13. R.E. Bellman, L.A. Zadeh, Decision-making in a fuzzy environment, Management science, 17 (1970) 141-164.

14. J. Qin, X. Liu, Multi-attribute group decision making using combined ranking value under interval type-2 fuzzy environment, Information Sciences, 297 (2015) 293-315.

15. W. Pedrycz, P. Ekel, R. Parreiras, Fuzzy multicriteria decision-making: models, methods and applications, John Wiley \& Sons, 2011.

16. W. Pedrycz, Allocation of information granularity in optimization and decision-making models: towards building the foundations of granular computing, $\mathrm{Eu}$ ropean Journal of Operational Research, 232 (2014) 137-145.

17. K.T. Atanassov, Intuitionistic fuzzy sets, Fuzzy sets and Systems, 20 (1986) 87-96.

18. K. Atanassov, G. Gargov, Interval valued intuitionistic fuzzy sets, Fuzzy sets and systems, 31 (1989) 343-349.

19. Z. Xu, R.R. Yager, Some geometric aggregation operators based on intuitionistic fuzzy sets, International journal of general systems, 35 (2006) 417-433.

20. $\mathrm{Z}$. $\mathrm{Xu}$, Intuitionistic fuzzy aggregation operators, IEEE Transactions on Fuzzy Systems , 15 (2007) 
1179-1187.

21. C.-P. Wei, P. Wang, Y.-Z. Zhang, Entropy, similarity measure of interval-valued intuitionistic fuzzy sets and their applications, Information Sciences, 181 (2011) 4273-4286.

22. W. Wang, X. Liu, Intuitionistic fuzzy information aggregation using Einstein operations, IEEE Transactions on Fuzzy Systems, 20 (2012) 923-938.

23. D.-F. Li, Mathematical-programming approach to matrix games with payoffs represented by Atanassov's interval-valued intuitionistic fuzzy sets, IEEE Transactions on Fuzzy Systems, 18 (2010) 1112-1128.

24. B. Farhadinia, A.I. Ban, Developing new similarity measures of generalized intuitionistic fuzzy numbers and generalized interval-valued fuzzy numbers from similarity measures of generalized fuzzy numbers, Mathematical and Computer Modelling, 57 (2013) 812-825.

25. J. Wu, F. Chiclana, A risk attitudinal ranking method for interval-valued intuitionistic fuzzy numbers based on novel attitudinal expected score and accuracy functions, Applied Soft Computing, 22 (2014) 272-286.

26. B. Liu, Y. Shen, W. Zhang, X. Chen, X. Wang, An interval-valued intuitionistic fuzzy principal component analysis model-based method for complex multi-attribute large-group decision-making, European Journal of Operational Research, 245 (2015) 209-225.

27. P. Liu, Some Hamacher aggregation operators based on the interval-valued intuitionistic fuzzy numbers and their application to group decision making, IEEE Transactions on Fuzzy Systems, 22 (2014) 83-97.

28. C. Tan, B. Ma, D.D. Wu, X. Chen, Multi-criteria decision making methods based on interval-valued intuitionistic fuzzy sets, International Journal of Uncertainty, Fuzziness and Knowledge-Based Systems, 22 (2014) 469-488.

29. S.-P. Wan, D.-F. Li, Fuzzy mathematical programming approach to heterogeneous multiattribute decisionmaking with interval-valued intuitionistic fuzzy truth degrees, Information Sciences, 325 (2015) 484-503.

30. X. Zhang, Z. Xu, Soft computing based on maximizing consensus and fuzzy TOPSIS approach to intervalvalued intuitionistic fuzzy group decision making, $A p$ plied Soft Computing, 26 (2015) 42-56.

31. B. Farhadinia, A theoretical development on the entropy of interval-valued fuzzy sets based on the intuitionistic distance and its relationship with similarity measure, Knowledge-Based Systems, 39 (2013) 79-84.

32. P. Liu, Some geometric aggregation operators based on interval intuitionistic uncertain linguistic variables and their application to group decision making, $A p$ plied Mathematical Modelling, 37 (2013) 2430-2444.

33. J. Wu, F. Chiclana, Non-dominance and attitudinal prioritisation methods for intuitionistic and interval- valued intuitionistic fuzzy preference relations, Expert Systems with Applications, 39 (2012)13409-13416.

34. Z.-J. Wang, K.W. Li, An interval-valued intuitionistic fuzzy multiattribute group decision making framework with incomplete preference over alternatives, Expert Systems with Applications, 39 (2012) 1350913516.

35. M. Grabisch, Fuzzy integral in multicriteria decision making, Fuzzy sets and Systems, 69 (1995) 279-298.

36. G. Choquet, Theory of capacities, in: Annales de l'institut Fourier, Institut Fourier, 1954, 131-295.

37. M. Sugeno, Theory of fuzzy integrals and its applications, Tokyo Institute of Technology, 1974.

38. R.R. Yager, Choquet aggregation using order inducing variables, International Journal of Uncertainty, Fuzziness and Knowledge-Based Systems, 12 (2004) 69-88.

39. F. Meng, X. Chen, Q. Zhang, Some interval-valued intuitionistic uncertain linguistic Choquet operators and their application to multi-attribute group decision making, Applied Mathematical Modelling, 38 (2014) 2543-2557.

40. Z. Xu, Choquet integrals of weighted intuitionistic fuzzy information, Information Sciences, 180 (2010) 726-736.

41. Z. Xu, Multi-person multi-attribute decision making models under intuitionistic fuzzy environment, Fuzzy Optimization and Decision Making, 6 (2007) 221-236.

42. C. Tan, A multi-criteria interval-valued intuitionistic fuzzy group decision making with Choquet integralbased TOPSIS, Expert Systems with Applications, 38 (2011) 3023-3033.

43. C. Tan, X. Chen, Intuitionistic fuzzy Choquet integral operator for multi-criteria decision making, $E x$ pert Systems with Applications, 37 (2010) 149-157.

44. F. Meng, J. Tang, Interval-Valued Intuitionistic Fuzzy Multiattribute Group Decision Making Based on Cross Entropy Measure and Choquet Integral, International Journal of Intelligent Systems, 28 (2013) 1172-1195.

45. F. Meng, H. Cheng, Q. Zhang, Induced Atanassov's interval-valued intuitionistic fuzzy hybrid Choquet integral operators and their application in decision making, International Journal of Computational Intelligence Systems, 7 (2014) 524-542.

46. Y. Xu, H. Wang, J.M. Merig, Intuitionistic fuzzy Einstein Choquet integral operators for multiple attribute decision making, Technological and Economic Development of Economy, 20 (2014) 227-253.

47. J. Wu, F. Chen, C. Nie, Q. Zhang, Intuitionistic fuzzyvalued Choquet integral and its application in multicriteria decision making, Information Sciences, 222 (2013) 509-527.

48. J.-q. Wang, D.-d. Wang, H.-y. Zhang, X.-h. Chen, Multi-criteria group decision making method based on interval 2-tuple linguistic information and Cho- 
quet integral aggregation operators, Soft Computing, 19 (2015) 389-405.

49. M. Xia, Z. Xu, N. Chen, Some hesitant fuzzy aggregation operators with their application in group decision making, Group Decision and Negotiation, 22 (2013) 259-279.

50. F. Meng, Q. Zhang, H. Cheng, Approaches to multiple-criteria group decision making based on interval-valued intuitionistic fuzzy Choquet integral with respect to the generalized $\lambda$-Shapley index, Knowledge-Based Systems, 37 (2013) 237-249.

51. R. Yang, Z. Wang, P.-A. Heng, K.-S. Leung, Classification of heterogeneous fuzzy data by Choquet integral with fuzzy-valued integrand, IEEE Transactions on Fuzzy Systems, 15 (2007) 931-942.

52. C. He, Approximation of polygonal fuzzy neural networks in sense of Choquet integral norms, International Journal of Machine Learning and Cybernetics, 5 (2014) 93-99.

53. M. Grabisch, E. Raufaste, An empirical study of statistical properties of Choquet and Sugeno integrals, IEEE Transactions on Fuzzy Systems, 16 (2008) 839850.

54. M. Timonin, Robust optimization of the Choquet in- tegral, Fuzzy Sets and Systems, 213 (2013) 27-46.

55. J.-L. Marichal, k-intolerant capacities and Choquet integrals, European Journal of Operational Research, 177 (2007) 1453-1468.

56. I. Kojadinovic, J.-L. Marichal, M. Roubens, An axiomatic approach to the definition of the entropy of a discrete Choquet capacity, Information Sciences, 172 (2005) 131-153.

57. S. Angilella, S. Greco, B. Matarazzo, Non-additive robust ordinal regression: A multiple criteria decision model based on the Choquet integral, European Journal of Operational Research, 201 (2010) 277-288.

58. J. Ashayeri, G. Tuzkaya, U.R. Tuzkaya, Supply chain partners and configuration selection: An intuitionistic fuzzy Choquet integral operator based approach, $E x$ pert Systems with Applications, 39 (2012) 3642-3649.

59. H. Bustince, M. Galar, B. Bedregal, A. Kolesarova, R. Mesiar, A new approach to interval-valued Choquet integrals and the problem of ordering in intervalvalued fuzzy set applications, IEEE Transactions on Fuzzy Systems, 21 (2013) 1150-1162.

60. Z. Wang, G. Klir, Fuzzy measure theory, Springer Science and Business Media, 2013. 